\title{
Wheat sRNA transcriptome reveals $R$-gene associated distinct temporal miRNA profiles in response to stem rust and leaf rust diseases
}

\section{Minu M Nair}

Central University of Kerala

Hari Krishna S Kumar

Central University of Kerala

Jyothsna S

Central University of Kerala

Krishna TS

Central University of Kerala

Manjunatha C

ICAR

\section{Sivasamy S}

ICAR

\section{Alagu Manickavelu ( $\nabla$ amanicks@cukerala.ac.in ) \\ Central University of Kerala https://orcid.org/0000-0003-2875-2290}

\section{Research Article}

Keywords: Wheat, stem rust, leaf rust, R-genes, miRNAs

Posted Date: June 8th, 2021

DOl: https://doi.org/10.21203/rs.3.rs-601768/v1

License: (9) This work is licensed under a Creative Commons Attribution 4.0 International License. Read Full License 


\section{Abstract}

Wheat rusts, the dreadful fungal diseases caused by members of the Puccinia genus remain as a major threat to global wheat production and food security. The $R$-gene mediated resistance has been employed as an efficient approach to develop rust resistant varieties. However, evolution of new fungal races and infection strategies put forward the urgency of unravelling novel genomics assisted molecular players, including non-coding RNAs for rust disease management. MicroRNAs are crucial regulators of genes associated with plant growth, development, abiotic and biotic stress responses. In this study, high throughput small RNA sequencing was performed on susceptible and resistant wheat near-isogenic lines inoculated with stem rust and leaf rust, together with mock inoculated controls. While miRNA mining in stem rust inoculated libraries revealed a total of twenty-six known and seven novel miRNAs, the search in leaf rust libraries culminated with twenty-two known and four novel miRNAs. The identified candidate miRNAs constituted both guide and passenger miRNA strands along with their isomiRs. Temporal expression pattern of differentially expressed miRNAs pinpoints their possible role during the progress of infection. Comparative analysis provides a better understanding of altered miRNA profiles associated with $\mathrm{Sr} 36$ and $L r 45$ disease resistance genes in response to stem rust and leaf rust, respectively. Also, the disclosure of guide strand, passenger strand and a variant of novel-Tae-miR02 with various sub-genomal origins might serve as an important common link between stem rust and leaf rust defense mechanisms downstream to the respective $R$-genes. Further, cis-regulatory elements that modulate MIR genes, targets of identified miRNAs related to various signal transduction pathways, and miR-SSRs which might be useful for rust disease control, are the emphasized attributes of our results. The outcome from this simultaneous study of miRNA profiles among two rust diseases and further characterization of the identified miRNAs can contribute to the expanding rust disease management programs.

\section{Introduction}

Wheat, a nutrient storehouse of diverse dietary essentials and an extensively cultivated crop plant is threatened by many biotic and abiotic constraints. Among them, wheat rust diseases caused by three species of Puccinia, a fungal pathogen, are dreadful diseases that trouble the grain quality, yield and induce increased disease management expenses[1]. The abrupt multiplication of rust spores with their ability to long distance dispersal via wind and their potent evolving nature to produce more dreadful pathotypes set wheat rust disease management at a higher risk[2]. Wheat rusts infect the plant parts above the ground, where the infected leaves or stem regions are characterized by rusty spots of infectious urediniospores. The three rust diseases encountered by wheat are; leaf rust caused by Puccinia triticina Eriks., stem rust caused by Puccinia graminis f. sp. tritici, and stripe rust caused by Puccinastriiformis $\mathrm{f}$. sp. tritici [3]. Stem rust, the most destructive agent spawned frightening famines in the past was once managed by introducing resistant wheat varieties. The emergence of new races of stem rust pathogen indicated the revival of this disastrous severe disease-causing threat to global wheat production, as almost all the commercial wheat varieties surrender to the newly evolved stem rust race[4]. Though leaf rust pathogen creates lesser menace during infection, their recurrent occurrence worldwide makes them a 
substantial agent causing the deprivation of annual wheat production than all the other rust pathogens[5]. Hence, there is an urgent stipulation for the comprehensive delineation of wheat rust epidemologies and their genetic basis for proper genomic approaches to produce rust-resistant wheat varieties.

Rust diseases can be effectively managed either by chemical methods or by genetic resistance[6]. Since genetic resistance has merit over the chemical methods due to reduced negative environmental impact and economically sound, attempts are made to determine the rust resistance mechanisms in wheat for long-lasting resistance[7]. The resistance gene, commonly known as $R$-gene is the primarily focused gene family in plant defense responses. The $R$-gene mediated genetic resistance can be predominantly classified into all stage resistance or seedling resistance and adult plant resistance[8]. Leaf rust and stem rust resistant genes are labelled as $L r$ and $S r$ genes, respectively[6]. Though each $R$-gene confers resistance through activation of different pathways, there is always ample conservation of defense mechanisms. These responses include production of reactive oxygen species (ROS), lipoxygenases and phytoalexins, cell wall fortification, activation of salicylic acid and jasmonic acid pathways, PR proteins, hypersensitive responses (HR) and defense related proteins, etc. [9]. The $R$-gene mediated responses are part of effector triggered immunity $(\mathrm{ETI})$, the secondary layer of plant immunity after pathogen associated molecular pattern (PAMP)-triggered immunity (PTI)[10]. Besides, the systemic acquired resistance (SAR) inductive capacity of $R$-gene mediated responses were also established during plantpathogen interactions[11]. The advent of identification and utilization of potent and durable resistance genes are found to be efficient in the head-to-head competition between wheat and rust pathogens. Introgression of R-genes such as Sr36 from Triticum timopheevi,Sr33 from Aegilops tauschii, Sr35 from T. monococcum etc., to polypoid wheat successfully imparted resistance against several stem rust strains, including the newly emerged devastating Ug99 strain[12][13]. Similarly, Lr45 from Secale cereale, Lr28 from Aegilops speltoides, Lr24 from Agropyran elongatum etc. were introgressed into wheat for providing effective leaf rust resistance[14][15][16]. The continuous evolution of stem rust and leaf rust races can overthrow these existing resistance genes. This pattern of sustained variations and increased hostility expressed by the rust pathotypes alarm the need for analyzing the unexplored downstream players in the defense response.

Exploring the complex regulatory mechanisms acting over $R$-genes, including those that involve regulatory non-coding RNAs (ncRNAs) are of prime importance in this context. Studies are interpreting the role of various ncRNA mediated molecular mechanisms allied with defense responsive activities in plants. In spite of having small size, small RNAs, especially microRNAs (miRNAs), have emerged as an important riboregulator that regulates the expression of their targets at post-transcriptional level[17]. Growing evidence revealed that these miRNAs, which are about 18-25 nt in length, exhibit differential expression during plant-pathogen interactions[18]. These miRNAs have been a major focusing area for a long time in plant growth, development, abiotic stress responses, plant-microbe interaction etc. The potential role of these (micro) players in mediating symbiotic relations, inducing disease susceptibility and providing disease resistance are well documented[18]. The ability of miRNAs to efficiently transform the expression of different targets at once, existence of conserved miRNA-mRNA pairs in significant plant 
regulatory networks and cross-kingdom regulation by these tiny regulators forge them as promising candidates for plant improvement. Many miRNA-based strategies such as designing artificial miRNAs, miRNA mimic, artificial target mimic etc. can be used when miRNAs regulate the gene of interest[19]. Over the years, over expression lines for miRNAs such as Osa-miR7696 conferred resistance to rice blast disease, miR393, miR160 and miR167 were reported for bacterial resistance and the list continues[20] [21]. The huge size of wheat genome, together with the differential spatio-temporal expression pattern of miRNAs hinder the precise identification of wheat miRNAs associated with particular traits[22]. The differential expression of miR159, miR164, miR167, miR171, miR444, miR408, miR1129 and miR1138 were a set of miRNAs found in stem rust infected wheat, which seems to have regulatory role over $R$ genes[23]. Recent studies depicted several pathogen-responsive miRNAs as fine regulators of resistance genes, especially nucleotide-binding site-leucine rich repeats (NBS-LRRs), pathogenesis-related (PR) proteins etc. [23][24]. However, alteration in miRNA profiling associated with resistance gene introgression and identification of regulators of precise miRNA production are poorly studied areas. This study aims to profile the conserved and novel miRNAs in resistant and susceptible wheat near-isogenic lines (NILs), in response to stem rust and leaf rust diseases using high-throughput small RNA sequencing. In addition, comparative analysis among the two-rust disease associated repertoire is performed since the sequencing libraries are made on a common susceptible background (HD2329) and two separate $R$ genes (Sr36 for stem rust and $L r 45$ for leaf rust) are introgressed on the same parent.

\section{Materials And Methods}

\subsection{Plant material and pathogen inoculation}

The HD2329 cultivar susceptible to both the rust diseases and it's NILs carrying Sr36 gene and Lr45gene were used as susceptible and resistant plants. The $S r 36$ and $L r 45$ carrying resistant plants were confirmed by gene specific markers Xstm77-3 and G372 respectively. Puccinia graminis f.sp. tritici pathotype 40A and Puccinia triticina pathotype 77-5 (the most predominant and devastating Indian rust pathotypes) were selected as experimental pathogens. Resistant and susceptible plants were inoculated with specific pathogen races that were suspended in Milli-Q water. Plants inoculated with Milli-Q water alone were used as mock inoculated control (Table 1). 


\begin{tabular}{|llll|}
\hline Disease & Wheat line & Inoculation treatments & Library name \\
\hline \multirow{3}{*}{ Stem rust } & HD2329 & Milli-Q (Mock) & SSM \\
\cline { 3 - 4 } & & 40A pathotype (Infection) & SSI \\
\cline { 3 - 4 } & HD2329+Sr36 & Milli-Q (Mock) & SRM \\
\cline { 3 - 4 } Leaf rust & HD2329 & M0A pathotype (Infection) & SRI \\
\cline { 3 - 4 } & & Milli-Q (Mock) & LSM \\
\cline { 3 - 4 } & HD2329+Lr45 & Milli-Q (Mock) & LRI \\
\cline { 3 - 4 } & & 77-5 pathotype (Infection) & LRI \\
\cline { 3 - 4 } & & &
\end{tabular}

Table 1. Wheat NILs and small RNA libraries. Stem rust and leaf rust infection treated and mock treated cultivars along with the designated library names are enlisted.

Leaf samples of two leaf stage from the treated plants were collected at 24 hours post inoculation (hpi) for library preparation. Samples from $0,6,12,18,24,36,48,72$ hpi were collected for qRT-PCR based miRNA expression studies. Infection symptoms were confirmed by visual examination of plants on 10 days post inoculation (dpi). Leaf samples were dipped in RNAlater (Qiagen) immediately, frozen and stored at $-80^{\circ} \mathrm{C}$ for RNA isolation. Growing plants, pathogen inoculum preparation followed by inoculation, and sample collection were performed at the glass house facilities, IARI, Wellington, by following the protocol mentioned in Singh et al., 2012[25].

\subsection{Construction of small RNA libraries and sequencing}

Total RNA was isolated using Trizol (Invitrogen), followed by the removal of genomic DNA by DNase (Catalog \# AM1906, Ambion), and examined by gel electrophoresis. Quality of total RNA was analysed by Agilent Tape station 2200 (Agilent Technologies). The RNA integrity number (RIN) was determined for all the samples before proceeding to library construction. $1 \mu \mathrm{g}$ of total RNA was taken and preceded for library preparation using Truseq small RNA library prep kit (Cat\# RS-200-0012) as per the manufacturer's instructions. Final libraries were quantitated by Qubit 2.0 fluorometer (Thermo Fisher Scientific) and the quality was analysed by Agilent Tape station 2200. Denatured libraries were single-end sequenced with 51 nts using the rapid mode of HiSeq 2500 (Illumina) v3 chemistry at the Institute of Bioinformatics and Applied Biotechnology (Banglore, India) following the vendor's recommended protocol.

\subsection{Analysis of sequence data}

The obtained raw reads were subjected to filtering of adapter, low quality reads, repeats and junk sequences to acquire the clean reads alone. Other common non-coding RNAs in the reads were identified and removed with the aid of Rfam database (http://rfam.sanger.ac.uk/). Reads of size $<18 \mathrm{nt}$ and $>30 \mathrm{nt}$ 
were discarded using Cutadapt tool [26]. Clean reads of all the libraries were mapped to Triticum aestivum (IWGSC release V2.0) genome using Bowtie and used for further analysis[27] [28].

\subsection{Identification of conserved and novel wheat miRNAs}

Reads mapped against Triticum aestivum were used to predict the novel and conserved miRNAs in wheat using miRDeep2 package[29]. To predict conserved miRNAs in wheat, all known plant miRNAs were used from miRBase release22[30]. Identified matches were named according to the most abundant miRNA isoform for the comparison. Identification of novel miRNAs was done using the criteria prescribed by Axtel et al., 2018 and the candidate novel miRNAs were filtered from miRDeep2 results accordingly[31].

The identification of miRNA variants (isomiRs) was done by mapping the sRNA reads to the miRNA hairpins of plant species available in miRBase, with no mismatch. The reads failed to map with them, were further mapped against the genome without any mismatches. The unmapped reads to genome were further remapped with plant hairpins with three mismatches[32]. The variants were manually detected with 5'-modifications, 3'- modifications and internal substitutions with the corresponding miRNAs.

\subsection{Promoter analysis of identified MIR genes}

To identify the chromosomal location of individual miRNAs, the precursor miRNA sequences were retrieved and mapped against the reference genome. Pre-miRNA sequence and upstream sequence up to $2 \mathrm{~kb}$ were retrieved after mapping the precursor sequences and used to discover cis-acting regulatory elements (CREs) in the MIR gene promoters. The Pol II promoter binding region and transcription start sites (TSS) were predicted with the TSSPlant web tool[33]. After the promoter prediction, a total of $500 \mathrm{nt}$ sequences (-400bp and $+100 \mathrm{bp})$ of the TSS were extracted and given as query for cis-element prediction in PlantCARE database[34]. The CREs with matrix score $\geq 7$ were retained for the study. We used SSRIT tool (http://www.gramine.org/db/searches/ssrtool) for mining miRNA-SSR markers from our data set, a $1000 \mathrm{bp}$ of primary miRNA sequence including the precursor miRNAs were used[35]. The di- and greater repeats with $n \geq 7$ were chosen.

\subsection{Analysis of differentially expressed miRNAs}

The differentially expressed miRNAs were identified using transcripts per million clean tags (TPM) and DESeq2 software[36]. Normalization of miRNAs were applied by TPM, using the following equation: TPM $=($ miR_readscounts $\times 1,000,000) /$ library size/ total counts of clean reads. After normalization, differentially expressed miRNAs were established by $\log 2$ value $>1$ and $<-1$ and a false discovery rate $(F D R)$ value $\leq 0.05$. VolcaNoseR generated volcano plots to visualize the differentially expressed miRNAs between libraries[37].

\subsection{Prediction of miRNA targets and functional analysis}

psRNATarget software was used to predict the target genes of miRNAs. The cDNA library Triticum aestivum (wheat), cDNA, Ensembl Plants release-43 was used with the scoring scheme V2 (2017 release) 
[38]. Target gene annotations were carried out by the Gene Ontology (GO) and KEGG pathway analysis in Blast2Go software[39]. The $\mathrm{GO}$ terms were further categorized according to their biological processes, molecular functions, and cellular components. The enriched metabolic pathways or signal transduction pathways of potential miRNA target genes were validated using KEGG enrichment analysis[40]. Networks between target genes of differentially expressed miRNAs were subsequently assembled using the String tool[41].

\subsection{Primer design, stem-loop RT- PCR amplification and quantification of miRNAs}

The stem-loop RT primers were designed manually as per the established protocol and their primers were designed by Primer3 (Table S1)[42][43]. The samples of SSM, SSI, SRM, SRI, LSM, LSI, LRM, and LRI at 0, $6,12,18,24,36,48,72 \mathrm{hpi}$ have opted for qRT-PCR. Total RNA was extracted using TRIZol. Reverse transcription for miRNA detection was performed using Superscript III reverse transcriptase (ThermoFisher 18080093). Real time PCR reactions of $20 \mu \mathrm{L}$ volumes were performed using iTaq universal SYBR Green master mix (BioRad 1725121) according to the manufacturer's protocol in the LightCycler 480 system (Roche Life Science). The wheat U6snRNAwas used as the internal control. All reactions were performed using one biological sample with three technical replicates. The gene expression levels were calculated according to the $2^{-\Delta \Delta C T}$ method as described[44].

\section{Results}

\subsection{High-throughput small RNA sequencing}

Altogether, eight sRNA libraries were created and sequenced upon mock inoculation and fungal inoculation. More than $90 \%$ clean reads were obtained after removing poly- $\mathrm{N}$, adaptor sequences, low quality reads, reads shorter than 18 and reads greater than 30 . The reads were enriched for wheat-specific sRNAs by mapping to the reference genome provided by IWGSC. The mapping efficiency of these libraries was between $27 \%$ and $80 \%$. Read statistics are given in Table 2 .

\begin{tabular}{|llll|}
\hline Library Name & Total reads & Clean reads & Mapped reads \\
\hline SSM/LSM & 13730752 & 13593253 & 10850834 \\
\hline SSI & 16949814 & 16742128 & 11786818 \\
\hline SRM & 17076363 & 16865615 & 4674895 \\
\hline SRI & 8581470 & 8460391 & 5936137 \\
\hline LRM & 16321187 & 16053416 & 9123654 \\
\hline LRI & 18922571 & 18734697 & 9271508 \\
\hline
\end{tabular}


Table 2. Read statistics of stem rust and leaf rust associated wheat libraries. Total reads obtained from sequencing, processed clean reads and mapped reads out of them are listed.

\subsection{Identification of known and novel wheat miRNAs}

Overall, we have identified twenty-six known miRNAs and seven novel miRNAs in the stem rust libraries and twenty-two known and four novel miRNAs for leaf rust libraries. Previously known miRNAs include the star miRNAs/ passenger strands and miRNA variants of known mature miRNAs. The miRNAs obtained from this study are listed in Table 3. 


\begin{tabular}{|c|c|c|c|}
\hline Library & miRNAs & Homologous miRNAs & miRNA seq \\
\hline SSM/ & miR9774 & Tae-miR9774 & caagaucuuggguauuucugucu \\
\hline \multirow[t]{10}{*}{ LSM } & miR1848 & Osa-miR1848 & ucgccggcgcgcgcgugcaucg \\
\hline & miR9776 & Tae-miR9776 & uggacgaggaugugcagcugc \\
\hline & miR159a & Tae-miR159a & uuuggauugaagggagcucug \\
\hline & miR168-3p & Hvu-miR168-3p & cccgccuugcaccaagugaau \\
\hline & miR166b & Hvu-miR166b & ucggaccaggcuucauucccc \\
\hline & miR169f-3p & Ata-miR169f-3p & ggcaaguccguccuuggcuac \\
\hline & miR160f-5p & Osa-miR160f-5p & ugccuggcucccugaaugcca \\
\hline & miR156h-3p & Zma-miR156h-3p & ugcucacugcucuuccugucauc \\
\hline & iso-miR9653b & Tae- miR9653b & ccguggccaaggucucuugaggcu \\
\hline & novel-Tae-miR01 & NA & ccacuaaaguuguugguc \\
\hline \multirow[t]{12}{*}{ SSI } & miR9774 & Tae-miR9774 & caagaucuuggguauuucugucu \\
\hline & miR166b & Hvu-miR166b & ucggaccaggcuucauucccc \\
\hline & miR160f-5p & Osa-miR160f-5p & ugccuggcucccugaaugcca \\
\hline & miR5384-3p & Tae-miR5384-3p & cgggaagcgegccgccgucg \\
\hline & miR9662b-3p & Tae-miR9662b-3p & ugaacaucccagagccaccgg \\
\hline & miR5048-5p & Tae-miR5048-5p & uauauuugcagguuuuaggucu \\
\hline & miR156e-5p & Bdi-miR156e-5p & ugacagaagagagugagcac \\
\hline & iso-miR9653b & Tae- miR9653b & ccguggccaaggucucuugaggcu \\
\hline & miR9669-5p & Tae-miR9669-5p & uacugugggcacuuauuugaca \\
\hline & miR396e-5p & Osa-miR396e-5p & uccacaggcuuucuugaacug \\
\hline & miR9776 & Tae-miR9776 & uggacgaggaugugcagcugc \\
\hline & miR9863a-3p & Ata-miR9863a-3p & ugagaagguagaucauaauagc \\
\hline \multirow[t]{5}{*}{ SRM } & miR9774 & Tae-miR9774 & caagaucuuggguauuucugucu \\
\hline & miR160f-5p & Tae-miR160f-5p & ugccuggcucccugaaugcca \\
\hline & iso-miR9653b & Tae- miR9653b & ccauggccaaggucucuugaggcu \\
\hline & miR5384-3p & Tae-miR5384-3p & cgggaagcgcgccgccgucga \\
\hline & miR168-3p & Hvu-miR168-3p & cccgccuugcaccaaguga \\
\hline
\end{tabular}




\begin{tabular}{|c|c|c|c|}
\hline & miR9655-3p & Tae-miR9655-3p & uggcuacuuccuuucccuugcc \\
\hline & iso-miR156d-3p & Ata-miR156d-3p & gcucacuccucuuucuguca \\
\hline & miR9662b-3p & Tae-miR9662b-3p & ugaacaucccagagccaccgg \\
\hline & miR169f-3p & Ata-miR169f-3p & ggcaaguccguccuuggcu \\
\hline & miR9776 & Tae-miR9776 & uggacgaggaugugcagcugc \\
\hline & iso-miR169r-3p & Zma-miR169r-3p & ggcaaguugucuuuggcu \\
\hline & miR166b & Hvu-miR166b & ucggaccaggcuucauucccc \\
\hline & novel-Tae-miR02-3p & NA & cccgcgcgcuacuccgucgag \\
\hline & novel-Tae-miR03 & NA & guaugagcccgguggacuagc \\
\hline & novel-Tae-miR04 & NA & cauggccaaggccucugaggucg \\
\hline \multirow[t]{17}{*}{ SRI } & miR9774 & Tae-miR9774 & caagaucuuggguauuucugucu \\
\hline & miR160f-5p & Osa-miR160f-5p & ugccuggcucccugaaugcca \\
\hline & miR168a-5p & Zma-miR168a-5p & ucgcuuggugcagaucgggac \\
\hline & miR169h-3p & Bdi-miR169h-3p & ggcagucaccuuggcuagc \\
\hline & $\operatorname{miR} 9662 b-3 p$ & Tae-miR9662b-3p & ugaacaucccagagccaccgg \\
\hline & miR398 & Tae-miR398 & uguguucucaggucgeccccg \\
\hline & miR168a-5p & Hvu-miR168a-5p & ucgcuuggugcagaucgggac \\
\hline & miR166b & Hvu-miR166b & ucggaccaggcuucauucccc \\
\hline & iso-miR9653b & Tae- miR9653b & ccguggccaaggucucuugaggcu \\
\hline & miR156d & Bna- miR156d & ugacagaagagagugagcac \\
\hline & novel-Tae-miR02-5p & NA & cucgccggagcagcaugucgu \\
\hline & miR1848 & Osa-miR1848 & ucgccggcgcgcgcgugcaucga \\
\hline & miR399e-3p & Zma-miR399e-3p & ugccaaaggagaguugcccug \\
\hline & miR169f-3p & Ata-miR169f-3p & ggcaaguccguccuuggcu \\
\hline & miR396c & Zma-miR396c & uccacaggcuuucuugaacug \\
\hline & miR9863a-3p & Ata-miR9863a-3p & ugagaagguagaucauaauagc \\
\hline & miR5384-3p & Tae-miR5384-3p & cgggaagcgcgccgccgucga \\
\hline \multirow[t]{2}{*}{ LSI } & miR9774 & Tae-miR9774 & caagaucuuggguauuucugucu \\
\hline & miR166b & Hvu-miR166b & ucggaccaggcuucauucccc \\
\hline
\end{tabular}




\begin{tabular}{|c|c|c|c|}
\hline \multicolumn{4}{|r|}{ ugccuggcucccugaaugcca } \\
\hline & miR156d-3p & Ata-miR156d-3p & gcucacuccucuuucugucagc \\
\hline & miR9662b-3p & Tae-miR9662b-3p & ugaacaucccagagccaccgg \\
\hline & miR393a-5p & Ata-miR 393a-5p & uccaaagggaucgcauugauc \\
\hline & miR393a-3p & Ata-miR 393a-3p & ucagugcaaucccucuggaau \\
\hline & iso-miR9653b & Tae- miR9653b & ccguggccaaggucucuugaggcu \\
\hline & miR167c-5p & Tae-miR167c-5p & ugaagcugccagcaugaucuga \\
\hline & miR167c-3p & Tae-miR167c-3p & aggucaugcuggaguuucauc \\
\hline & miR9776 & Tae-miR9776 & uggacgaggaugugcagcugc \\
\hline & miR5384 & Tae-miR5384 & cucgecggucgegcguccucccu \\
\hline & miR168-3p & Hvu-miR168-3p & cccgccuugcaccaagugaau \\
\hline & novel -Tae-miR07 & NA & ccgacccgaacgaaaggcga \\
\hline & novel -Tae-miR08 & NA & ccuguagaauucccggguccca \\
\hline \multirow[t]{4}{*}{ LRM } & miR9774 & Tae-miR9774 & caagaucuuggguauuucugucu \\
\hline & miR160f-5p & Tae-miR160f-5p & ugccuggcucccugaaugcca \\
\hline & $\operatorname{miR9662a-3p}$ & Tae-miR9662a-3p & uugaacaucccagagccaccg \\
\hline & miR166b & Hvu-miR166b & ucggaccaggcuucauucccc \\
\hline \multirow[t]{12}{*}{ LRI } & miR9774 & Tae-miR9774 & caagaucuuggguauuucugucu \\
\hline & miR160f-5p & Osa-miR160f-5p & ugccuggcucccugaaugcca \\
\hline & miR168a-5p & Hvu-miR168a-5p & ucgcuuggugcagaucgggac \\
\hline & miR9776 & Tae-miR9776 & uggacgaggaugugcagcugc \\
\hline & miR9662b-3p & Tae-miR9662b-3p & ugaacaucccagagecaccgg \\
\hline & miR9655-3p & Tae-miR9655-3p & uggcuacuuccuuucccuugcc \\
\hline & miR159a & Tae-miR159a & uuuggauugaagggagcucug \\
\hline & miR166b & Hvu-miR166b & ucggaccaggcuucauucccc \\
\hline & iso-miR9653b & Tae- miR9653b & ccguggccaaggucucuugaggcu \\
\hline & miR156d-3p & Ata-miR156d-3p & gcucacuccucuuucugucagc \\
\hline & iso-novel-Tae-miR02-3p & NA & cccgcgcgcuacuccgucgagc \\
\hline & miR1848 & Osa-miR1848 & ucgccggcgcgcgcgugcaucga \\
\hline
\end{tabular}


Table 3. Distribution of miRNAs in the stem rust and leaf rust libraries. The miRNAs are classified into conserved and novel. Novel miRNAs and isomiRs are represented with prefix 'novel-Tae' and 'iso' respectively. The suffix $-5 p$ and $-3 p$ refers to guide and passenger strands respectively.

The most and least number of miRNAs were produced from SRI and LRM libraries, respectively. While a greater number of miRNA variants/ isomiRs were produced from SRM, both SRM and SRI library had the highest number of novel miRNAs. Besides, no novel miRNAs were induced from SSI and LRM inoculations. Moreover, we also observed the generation of a novel mature miRNA novel-Tae-miR02-5p in SRI, it's passenger strand (novel-Tae-miR02-3p) in SRM and its variant iso-novel-Tae-miR02-3p in the LRI library. Interestingly, pairs of the guide strand (mature miRNA) and their corresponding passenger strands (miRNA*) for three MIR genes were present in three libraries. miR393a-5p - miR393a-3p pair and miR167c$5 p-$ miR167c-3p pair were present in the LSI, and miR169f-5p and miR169-3p was present in both SSM and LSM (which are replicates of mock treated HD2329 parental plants). We observed low abundant smaller size variants of two known miRNAs with 5' modifications, 3' modifications and internal substitutions in SRI and LRM treatments. In SRI library, a variant of Pab-miR11418 (variant: CCCGCCGCCGCCGCCGCCGC) contained variants with one nucleotide trimmed from $5^{\prime}$ and two internal substitutions, whereas the LRM contained a variant of Osa-miR5281 (variant: AGGGACGGAGCGAGGGU) with two nucleotides trimmed from $5^{\prime}$ end and one internal substitution. Length distribution of miRNAs was consistent with previous studies, where most of them are of $21 \mathrm{nt}$ length, and the 5 ' position is preferred for Uracil (Figure S1).

Comparison among the libraries for specific diseases helped us to obtain common and distinct miRNAs. While miR9774, miR9662b-3p, miR166b and iso-miR9653b were found in common in the stem rust associated libraries, miR9774, miR160f-5p and miR166b were the common ones present among all the leaf rust libraries (Figure S2). A prominent number of exclusively expressing miRNAs were found in SRI and LSI libraries respectively for the two diseases. Comparison between the specific disease sets provides the glimpse on reshaping the miRNA repertoire due to the inclusion of particular $\mathrm{R}$ genes. The difference in miRNA repertoire between SSM and SRM as well as between SSI and SRI were on account of the absence and existence of the $\mathrm{Sr} 36$ gene. Admittance of $L r 45$ gene is responsible for the divergence in miRNA profiles between LSM and LRM together with LSI and LRI. It manifests that adding a single gene on the background can result in the inclusion and bypassing of several miRNAs.

\subsection{Chromosomal mapping of MIR genes}

The MIR gene mapping suggested that the $D$ sub-genome has generated an extremely high number of miRNAs (Figure 1, Figure S3). In particular, a greater number of miRNAs associated with both the diseases are from chromosome 6D. The 3B chromosome exhibited the least miRNA production. A few miRNAs were triggered from single, specific location, like miR9774, generated from 7D chromosome in all 
libraries. Concurrently, there were miRNAs originated from multiple locations. For example, miR166b, present in every single library, was produced from 5B, 5D,5A and 4D at once.

\subsection{Analysis of cis-regulatory elements in MIR gene promoters}

In an attempt to document the regulatory elements of MIR genes, fifty-eight different cis-regulatory motifs were identified from the obtained promoters. An extensive number of discrete motifs were found to be involved in light responsiveness. The remarkably enriched motifs were TCA element related to salicylic acid responsiveness, ABRE for abscisic acid responsiveness, AAGAA motif, WUN motif, 02 element of zein metabolism, GARE element involved in gibberellic acid responsiveness, TCCC element for light responsiveness etc. miR5384 of the LSI library has the maximum number of distinct CREs (Table S2).

\subsection{Mining of miRNA-SSRs}

In particular, eight SSR motifs were found in association with the miRNAs from all our libraries (Table 4). 


\begin{tabular}{|c|c|c|}
\hline Library & miRNAs & $\operatorname{SSR}(n)$ \\
\hline SSM/ & miR166b & gat (7) \\
\hline \multirow[t]{3}{*}{ LSM } & & tc (8) \\
\hline & & ga (12) \\
\hline & miR156h-3p & ggct (7) \\
\hline \multirow[t]{6}{*}{ SSI } & miR166b & gat (7) \\
\hline & & tc (8) \\
\hline & & ga (12) \\
\hline & miR160f-5p & tcct (7) \\
\hline & miR5384-3p & ct (7) \\
\hline & miR168a-5p & ac (8) \\
\hline \multirow[t]{5}{*}{ SRM } & miR166b & gat (7) \\
\hline & & tc (8) \\
\hline & & ga (12) \\
\hline & miR160f-5p & tcct (7) \\
\hline & miR5384-3p & ct (7) \\
\hline \multirow[t]{8}{*}{ SRI } & miR166b & gat (7) \\
\hline & & tc (8) \\
\hline & & ga (12) \\
\hline & miR160f-5p & tcct (7) \\
\hline & miR5384-3p & ct (7) \\
\hline & miR168a-5p & ac (8) \\
\hline & miR399e-3p & $\operatorname{ttc}(8)$ \\
\hline & miR396c & ct (7) \\
\hline \multirow[t]{4}{*}{ LSI } & miR166b & gat (7) \\
\hline & & tc $(8)$ \\
\hline & & ga (12) \\
\hline & miR160f-5p & tcct (7) \\
\hline LRM & miR166b & gat (7) \\
\hline
\end{tabular}




\begin{tabular}{|lll|} 
& & tc (8) \\
& & ga (12) \\
\cline { 2 - 3 } LRI & miR160f-5p & tcct (7) \\
& miR166b & gat (7) \\
& tc (8) \\
& ga (12) \\
\hline miR160f-5p & tcct (7) \\
\hline miR168a-5p & ac (8) \\
\hline
\end{tabular}

Table 4. SSRs associated with stem rust and leaf rust responsive host miRNAs. The number of repeating units are denoted as ' $n$ '.

It was found that the primary sequence of seven out of the total identified miRNAs had one or more SSR motifs. In our study, di-nucleotide repeats were abundant than tri- and tetra-nucleotide repeats. The range of repeats was varied from seven to seventeen for di-, seven to eight for tri- and seven for tetra-nucleotide motifs. All the SSRs predicted in the study were linked with conserved miRNAs. The SRI treatment was found to have the highest number of distinct miRNAs with the repeats. The most distinct number of repeats were present in the primary sequence of miR166b. Furthermore, same di-nucleotide motif, 'ct' was repeated for miR5384-3p and miR396c, yet the repeat number was different. 'ct' motif was repeated seventeen times in miR5384-3p and for seven times in miR396c.

\subsection{Differential expression profiling of miRNAs}

Twelve miRNAs were found to be differentially expressed between SSI and SSM libraries. Sixteen miRNAs were differentially expressed in SRI v/s SRM, fifteen in SRM v/s SSM and eighteen in SRI v/s SSI. Meanwhile, the number of differentially expressed miRNAs among leaf rust associated libraries were thirteen, eight, ten and nineteen in LSI v/s LSM, LRI v/s LRM, LRM v/s LSM and LRI v/s LSI, respectively (Figure 2, Figure S4).

The miRNAs that showed superlative differential expression were miR168a-5p of SRI v/s SRM (up regulated), miR169f-5p of SSI v/s SSM (down regulated) in stem rust, and that of leaf rust inoculations were 160f-5p (up regulated) and 9662b-3p (down regulated) of LRI v/s LSI. Certain miRNAs like miR168a5p, miR398, miR399-3p and novel-Tae-miR02-5p were upregulated parallelly in SRI v/s SRM and SRI v/s SSI. In the same way, miR169f-5p and miR160f-5p were down regulated both in SSI v/s SSM and SRM v/s SSM. Further, miR156d-3p, miR5048-5p, miR9655-3p, novel-Tae-miR04 and iso-novel-Tae-miR02-3p were upregulated in both LRI v/s LRM and LRI v/s LSI. In the same groups, miR160f-5p was found to be down regulated. Additionally, miR169f-5p, miR156h-3p and novel-Tae-miR01 were found to be up regulated and miR160f-3p was appeared to be down regulated in LSI v/s LSM and LRM v/s LSM. Most differentially expressed miRNAs were selected and validated using real time PCR. The stem loop RT-PCR 
followed by real time PCR was used to authenticate the differential expression of miRNAs. Though these miRNAs showed a similar pattern in expression between libraries as that of sRNA sequencing, they varied in fold change of expression in real time PCR with respect to sequencing results (Figure S5).

\section{7 miRNA target prediction and construction of their regulatory networks}

Multiple miRNAs found to target specific genes in rust treated plants. It was well established that a vast majority of identified miRNAs function through targeted degradation than translational repression. Predicted target genes for respective miRNAs of individual treatments are given in the circos plot (Figure 3, Table S3).

Highly enriched targets for the known miRNAs include homeobox-leucine zipper proteins (HOX33, HOX32, HOX9, HOX29), rolled leaf2, auxin response factors (ARF13, ARF8, ARF18, ARF22), transcription termination factors (mTERF2, mTERF8, mTERF9, mTERF15), Peroxidase 12, disease resistance proteins (RPM1, RGA2, RPP13), F-box proteins, proline-rich receptor-like protein kinase PERK1, cryptochrome 1a, transcription factor RAX2, protein DETOXIFICATION 49, growth-regulating factors (GRF1, GRF2, GRF10), chloroplastic crocetin glucosyltransferase, etc. Meantime, the novel miRNAs were found to target transcription factors (ICE1, bHLH162), anthocyanidin 5,3-0-glucosyltransferase, LRR receptor-like serine/threonine-protein kinase GS01, F-box/kelch-repeat protein SKIP11, protein argonaute MEL1, putative disease resistance proteins (RGA1, RGA3), aspartic proteinase nepenthesin-1, phenolic glucoside malonyl transferase 1, flavanone 3-hydroxylase, MLO-like protein 13 etc. Both guide and passenger miRNA strands present in the same library seems to have distinct targets. The miR167c-5p of LRI targets F-box proteins, 1-aminocyclopropane-1-carboxylate oxidase isoform, ER membrane protein complex subunit 6 , probable anion transporter 3 etc. whereas, miR167c-3p regulates DIMBOA UDPglucosyltransferase BX8, putative disease resistance protein RGA3, protein LUTEIN DEFICIENT 5, heparanase-like protein 1 etc. Similarly, miR393-5p of the same inoculation modulate the expression of transport inhibitor response 1-like protein, protein CLP1 homolog, putative cyclic nucleotide-gated ion channel 15, electron transporter thiol-disulfide exchange intermediate etc. At the same time, the passenger strand miR393-3p controls putative E3 ubiquitin-protein ligase RING1a, Cyclin-T1-4 etc.

GO analysis and KEGG pathway analysis demonstrated that most of the target genes were associated with plant defense, plant growth, development, basal metabolism, and hormonal signaling. Most enriched GO terms were found to be DNA binding, metal ion binding, ATP binding, protein serine threonine kinase activity, hydrolase activity in the molecular function category and transcription regulation, membrane transport, protein phosphorylation, oxidation-reduction process, auxin activated signaling pathway, defense response etc., in biological process (Fig. S7). The interaction between target genes of differentially expressed miRNAs revealed the involvement of targets in aminoacyl t-RNA synthesis pathway, propionate metabolism pathway, mRNA surveillance pathway, valine, leucine, isoleucine degradation pathways etc.

\subsection{Temporal expression analysis of miRNAs}


To understand how the expression of differentially expressed miRNAs alter with infection stages and mock inoculation, we measured the relative temporal expression of miRNAs from 6, 12, 18, 24, 36, 48 and $72 \mathrm{hpi}$ leaf samples compared to the $0 \mathrm{hpi}$ by real time PCR. In the mock treated libraries of stem rust disease (SSM and SRM), the differentially expressed miRNAs didnot show major shift in the expression with time. Instead, they had a maximum fold change up to four and exhibited minimal expression compared to the $0 \mathrm{hpi}$, at all other instances. Other than novel-Tae-miR03 with maximum activity at 18 hpi, every selected miRNA of SSM and SRM showed peak activity at $6 \mathrm{hpi}$, declined at later stages and marginally rebounded at intervals. In stem rust pathogen inoculated NILs, miRNAs seldom display any immense change in relative expression, except miR398 of SRI. miR156e-5p of SSI showed an increase in relative expression from $6 \mathrm{hpi}$ to a maximum at $24 \mathrm{hpi}$ and decreased past that time point. For miR396e$5 p$, the relative expression dropped from its maximum at $6 \mathrm{hpi}$ till $48 \mathrm{hpi}$ and later showed a slight increment at $72 \mathrm{hpi}$. miR9669-5p of SSI showed minimal expression at all time points with a drop at 36 and $48 \mathrm{hpi}$. Interestingly, relative expression of all the selected miRNAs of SRI extensively constrained after $36 \mathrm{hpi}$. Here, miR396c, miR398 and novel-Tae-miR06 showed peak expression at $6 \mathrm{hpi}$, miR9863a-3p and novel-Tae-miR0 5 expressed maximum at 36 hpi whereas, novel-Tae-miR02-5p exhibited maximum activity at $12 \mathrm{hpi}$.

Resembling mock inoculated libraries of stem rust disease, miRNAs of leaf rust libraries also showed minuscule variation in fold change at various temporal points. In LRM, miR160f-5p showed maximum activity at 6 hpi whereas, it was 12 hpi for miR166b. However, the differentially expressed miRNAs of infected samples exhibited an enormous shift in the fold change of expression, and individual miRNAs displayed peculiar profile with distinct maximum activity period. Within LSI library, miR156d-3p, miR166b and novel-Tae-miR07 had maximum activity at 6 hpi, the same for miR393a-5p, miR167c-5p and novelTae-miR08 were 18, 24 and 36 hpi, respectively. The miR167c-5p exhibited a relatively highest fold change in expression. The same miRNA gradually showed a hike in relative expression till $18 \mathrm{hpi}$, followed by a tremendous increment at $24 \mathrm{hpi}$, tumbled and maintained near to constant level of expression from $36 \mathrm{hpi}$ onwards. miR156d-3p, excluding their maximum activity period, comparably exhibited lower fold change with a slight shoot up at 18 and 48 hpi. miR393a-5p displayed nominal expression till $12 \mathrm{hpi}$, inflated to a maximum at $18 \mathrm{hpi}$ and gradually decreased with time. Whereas, novelTae-miR07 had a considerable drop in fold change after $6 \mathrm{hpi}$, then steadily increased up to $24 \mathrm{hpi}$ and then diminished with time. The same expression trend has been observed in miR166b till $24 \mathrm{hpi}$ and subsequently showed a reduced expression. miRNAs in LRI library had maximum fold change at $6 \mathrm{hpi}$, irrespective of their disparate expression profiles. miR156d-3p of LRI showed a very similar expression pattern to that of LSI. In addition, iso-novel-Tae-miR02-3p and novel-Tae-miR04 exhibited an identical temporal expression profile. Relative expressions of both miRNAs mentioned above declined from $6 \mathrm{hpi}$ to $18 \mathrm{hpi}$ and were found to surge from $24 \mathrm{hpi}$ to $48 \mathrm{hpi}$ followed by a drop in expression level as time proceeds.

\subsection{Comparative analysis of miRNA profiles identify novel-Tae-miR02 as a link between stem rust and leaf rust resistance interaction}


The uniqueness and distinctiveness of miRNA profiles among the two rust disease treatments could be obtained through their comparison. Generation of distinct known and novel miRNAs were profound in stem rust associated libraries. On a correlative note, miR9774 and miR166b were present among all the libraries, irrespective of the inoculation. The stem rust pathogen inoculation on the susceptible and resistant cultivars bred miRNAs from miR396 family, whereas leaf rust infection lacks any such miRNA generation. Interestingly, closely related novel miRNAs were induced in both the resistant plants upon various inoculations. It was novel-Tae-miR02-5p of SRI, its passenger strand novel-Tae-miR02-3p in SRM and its single nucleotide variant iso-novel-Tae-miR02-3p in LRI. Further analysis on precursors of these miRNAs showed that novel-Tae-miR02-5p was produced from A sub-genome with different chromosomal locations such as $2 \mathrm{~A}, 5 \mathrm{~A}$ and $7 \mathrm{~A}$, whereas, its passenger strand and its isomiR were originated from $1 \mathrm{D}$ or the $D$ sub-genome. The passenger strand of novel-Tae-miR02 (novel-Tae-miR02-3p) of the A-sub-genome has variations from $D$ sub-genome derived novel-Tae-miR02-3p, though the novel-Tae-miR02-5p strands are identical in both the sub-genome.

Further, multiple sequence alignment was performed with the precursors of $A$ and $D$ sub-genome derived novel-Tae-miR02 and observed $\sim 80 \%$ identity between the two precursors with a cladogram branch length of 0.208 (Fig. S8). It was interesting to spot the co-occurrence of novel-Tae-miR04 with novel-TaemiR02-3p in SRM and iso-novel-Tae-miR02-3p in LRI inoculation treatments. The specific gene family was found as a shared target among novel-Tae-miR02-5p, novel-Tae-miR02-3p, and the variant iso-novelTae-miR02-3p was expansin have diverse functions in physiological and environmental responses in plants. Apart from the communal targets shared with the variant, novel-Tae-miR02-3p had supplementary targets include endo-1,4-beta-xylanase Z, magnesium transporter MRS2-E-like, trihelix transcription factor ASR3-like and GATA transcription factor 8-like.

\section{Discussion}

Though many miRNAs have been identified in wheat, the list is not saturating, and their profiling at individual conditions will provide new paradigms of their functions. Also, the rapid lineage specific evolution of plant miRNAs complements the incessant possibility of finding novel miRNAs. The noncoding RNA mediated regulation of plant disease resistance genes have been a thrust area of research for a long, even so, how the resistance genes affect miRNA generation in the defense response is not yet elucidated. So, the main goals of this study were to investigate the potential miRNA repertoire during resistant as well susceptible interaction of stem rust and leaf rust with wheat and establish how the Rgene introgression alters the miRNA profiles in parental plants with and without pathogen infection. For that motive, two of the all-stage resistance genes namely Sr36 and Lr45 introgressed into a common susceptible background were used for miRNA profiling. The miRNA production was high upon fungal ingress compared to their corresponding mock inoculations. The identified miRNAs include the guide strands, passenger strands, and miRNA variants of conserved and novel miRNAs. Though the miRNA star strands/ passenger strands are established as independent gene regulators, they can also co-regulate their targets along with their corresponding guide strands[45][46]. This study exposes three pairs of miRNA guide and passenger strands co-occurrence, which include miR169f-5p and miR169f-3p of 
SSM/LSM, miR 167c-5p miR167c-3p pair together with miR 393a-5p - miR393a-3p pair in LSI. The synchronised targeting of different genes by miR393 (same family of miR393a) and its passenger strand miR393* were previously reported in Arabidopsis infected with the bacterial pathogen Pseudomonas syringae pv. tomato (Pst)[47][48]. Apart from some low abundant miRNA variants, most of the other variants identified in the study exhibited abundance similar to real miRNAs. These miRNA variants are designated as isomiRs, which probably belong to the same miRNA family but varied in length, likely due to $5^{\prime}$ or $3^{\prime}$ modifications and may sometimes exhibit nucleotide substitutions as well[49]. These variants were earlier considered as imprecise products of miRNA processing, and now established to have role in regulating genes in a miRNA-like manner[50]. Most of the stem rust and leaf rust associated miRNAs were $21 \mathrm{nt}$ in length. In general, the molecular rulers for plant miRNA length are the DCL proteins, where DCL1 is linked with the production of 21nt long plant miRNAs[51].

Similarly, the preponderance of identified miRNAs with Uracil at the 5' position may refer to the activity of AG01 protein that prefers miRNA cargoes with 5' Uracil[52] which indicates the leading role of DCL1 and AG01 mediated processing of miRNAs in response to these diseases. The miRNAs present in all the inoculation treatments of the particular disease might indicate their role in that specific developmental stage of the plant. Moreover, the novel miRNAs obtained were affirmed by separate level of testimonies like, appropriate secondary structure, folding energy parameters, mapping pattern of mature strands on the precursor, mismatch with the passenger strands, real time PCR based expression etc. Both Sr36 and Lr45 introgression undoubtedly produced huge change in miRNA repertoire compared to their parental line HD2329. In a study by Jain et al., 2020, the differential miRNA and long non-coding RNA (IncRNA) expression were presented from HD2329 cultivar and its NIL of Lr28[53]. Similarly, differential miRNA expression between HD2329 and its NIL with Lr24 was studied earlier[24]. These studies confirmed the possibility of drastic changes in miRNA generation with the addition of a single $R$-gene in the parental plant.

The hexaploid nature of wheat justifies the presence of more copies of miRNAs from multiple locations in the genome. The sub-genome dynamics for miRNA generation disclosed that a higher number of miRNAs were from relatively younger $D$ sub-genome, which may attribute to the latest hybridization event of wheat with the $\mathrm{D}$ genome donor. Though miRNAs are documented as gene expression regulators, the spatio-temporal control over the differential expression of these miRNAs remain as a less explored area. Various transcription factors (TFs) modulate MIR genes, like the case of protein coding genes[54][55]. So, identifying TFs with binding sites on the precursor miRNA will provide an overviewof regulation of specific miRNA genesis. The identified MIR promoters were enriched with CRE elements for light responsiveness. In plants, the capacity of light in regulating MIR transcription, host miRNA biogenesis, RISC loading, differential expression and their downstream biological activity were previously established[56]. Specifically, the circadian clock can modulate non-coding RNAs similar to coding genes and correlate with the circadian motif found in miR156d-3p of LRI library[57]. The CREs related to the response of salicylic acid, abscisic acid, gibberellin, jasmonic acid, auxins etc. shows the favourable effects of these miRNAs in plant growth, development, and stress responses[58]. 
Further, GCN4 and G-box coexisted in the promoters of miR168a-5p in SSI, SRI and LRI are related to ROS generation, which might have an association with defense responses[59]. In addition, TGACG motif, ERE element, ethylene responsive elements, $A B A$ responsive elements and $S A$ responsive elements were also reported to contribute oxidative stress responses[60][61][62]. Circadian, 02 site and MBSI were involved in cellular development, apart from the role of $\mathrm{O} 2$ site in zein metabolism and positive role of MBSI during flavonoid biosynthesis. The ABRE and DRE elements were regulated by calcium responses[58].

The SSR markers, co-dominant molecular markers, play an inevitable role in molecular evolution studies[63][64]. The miRNA-SSRs are extremely useful as functional markers for identifying genetic diversity of miRNAs and developing specific trait associated selection programs due to their conditionspecific expression[65]. However, the number of discovered plant miRNA-SSRs are very limited compared to their immense applications. The repeat associated with miR168a-5p was there in SSI, SRI, and LSI and found to be a promising candidate. Also, SSR associated with miR399e-3p could be a marker for stem rust resistance interaction due to their specificity in the SRI treatment. The identified miRNA-SSRs might have the extensive potential for crop improvement associated with rust management in wheat. The pattern of differential miRNA expression was consistent among sequencing and real time PCR, yet the value of fold change was less in qRT-PCR, which can be accounted for the variation in sensitivity between instruments of high throughput sequencing and real time PCR.

Most of the predicted targets of miRNAs in this study are necessary for the growth and development of plants, indicating the possibility of compromising basal metabolic events at the cost of disease management. Many of these identified miRNA-target pairs were previously reported. The miRNAs belonging to miR9662 family targeted mitochondrial transcription termination factor, mTERF15 in bread wheat[66]. They also speculate that miR9662a-3p might be a vital modulator of wheat growth and development since their expression is damped with the plant's growth. In addition to mTERF15, other mTERFs like mTERF2, mTERF8 and mTERF9 were also predicted as target genes of miR9662a-3p and miR9662b-3p in the treatments. mTERFs, localised in mitochondria and chloroplast, showed their inevitable roles in transcription initiation, DNA replication, organ development, etc. [67][68]. Members of miR396 family were produced specifically in the stem rust infected NILs and targeted TFs belonging to GRFs. miR396 family mediated modulation of GRFs, which are important for growth and stress response, was evolutionally conserved across many plant species[69][70][71][72]. Recently, the activity of miR396 was reported to negatively regulate host immunity upon biotrophic and hemi-biotrophic infection in Arabidopsis[73]. Here miR396 mediates reprogramming of biological processes such as defense response, cell wall modification, cytokinin synthesis, pathway regulation etc., by targeting GRFs. Induction of miR396 members in stem rust infected plants could be possibly controlled by the pathogen.

Similarly, members of miR160 mediated targeting of ARFs were previously established[74][75][76]. In potato, miR160 was identified as an important regulator of defense responses and systemic acquired resistance upon phytophthora infections[77]. Similar to this study, targeting TF MYB by miR159 and class III homeobox leucine zipper proteins by miR166, associated with downy mildew fungal infection, were reported in cucumber[78]. In the present study, novel-Tae-miR08 of LSI was found to target two specific 
cysteine-rich receptor-like protein kinases (CRKs); CRK6 and CRK25. The CRKs belong to a large subfamily of plant receptor like kinases (RLKs) capable of signal perception[79]. Various representatives of CRKs explicitly respond to plant pathogens and PAMP treatments[80]. An investigation in Arabidopsis disclosed that overexpression of $C R K 6, C R K 4$ and $C R K 36$ strengthened the activation of PTI responses and boosted the resistance to Pst[81]. In addition, a recent study discovered a novel CRK gene, TaCRK2 as a positive regulator of wheat resistance to Puccinia triticina infection[82]. The TaCRK2 gene, related to calcium signalling was upregulated in an incompatible interaction with $P$. triticina race 260 and $L r 26$ introgressed wheat. The positive role in leaf rust resistance was confirmed by overexpression and knockdown studies of TaCRK2 gene[82]. The current study speculates that novel-Tae-miR08, contribute to leaf rust disease susceptibility through inactivation of CRK6 and CRK25. Furthermore, novel-Tae-miR04 identified both in SRI and LRM targets AT-hook motif nuclear-localized protein 27 (AHL27). AHL27 is a TF that reported to bind AT-rich sequences linked to nuclear matrix attachment regions[83]. Studies found that $A H L 27$ negatively regulates PTI responses to pathogens via suppression of PAMP triggered FRK1 expression[84]. Similarly, other members of the family $A H L 19$ and $A H L 15$ also abolish PAMP-induced gene expression. Additionally, over expression lines of $A H L 20$ showed improved resistance against $P$. syringae infection in Arabidopsis[84]. Here, novel-Tae-miR04 may enhance resistance responses by negatively regulating the suppressor of PTI responses. Generally, a vast majority of stem rust and leaf rust responsive miRNAs in this study, regulate TFs and disease resistance proteins. Two major possible reasons for regulation of disease resistance genes within resistant plants are either hijacking of the same and their signalling pathways by the fungal pathogen or as the host mechanism to control constitutive production of these proteins which have detrimental effects on growth and development of the host, especially in younger stages [85].

The current research also delves into the temporal dynamic expression pattern of identified miRNAs since the pattern of plant miRNA expression alters dramatically as the fungi proceed to different infection stages with time[86]. Time series expression study of miRNAs of individual inoculation was done to evaluate the dynamics of individual miRNAs at various conditions. The strongest responses of individual miRNAs at particular treatment were identified by analysing peak activity period. Most of the miRNAs in mock inoculations exhibited minimal expression, which may be associated with the plant's growth stage. Generation and minimal expression of miRNAs in the mock inoculated resistant NILs could be attributed to the metabolic changes in the host associated with the inclusion of corresponding $R$-genes. The evident relative expression of miRNAs of SRI library till $36 \mathrm{hpi} \mathrm{might} \mathrm{directly} \mathrm{link} \mathrm{to} \mathrm{the} \mathrm{activity} \mathrm{of} \mathrm{Sr36.} \mathrm{The}$ resistance response mediated by the aforementioned $R$-gene is through callose deposition at the very early stage of infection. Due to their quick activity, haustoria formation infrequently occurs in Sr36 lines[87]. The expression of identified miRNAs in pathogen infected Sr36 carrying lines of the current study also correlated with this fact. In general, the Puccinia fungal urediniospores germinate hours after infection. As the infection proceeds, appressorium for infection spread and haustorium for intimate feeding relationship seems to establish by $8 \mathrm{hpi}$ and $24 \mathrm{hpi}$, respectively. From the $48 \mathrm{hpi}$ onwards, secondary infection within the plants begins [88]. Hence, highly variable activities of specific miRNAs identified in this study can be associated with their role in different stages of fungal infection. On a 
decisive note, miRNAs at 6 hpi might be associated with appressorium formation, until 24 hpi might be related to haustorium formation and past to that might possibly be associated with secondary infection in rust-treated libraries. In addition, specific miRNAs such as miR156d-3p, novel-Tae-miR04 etc. with diverse temporal expression pattern on different inoculation treatments indicates the prospect of various regulatory functions and extent of limiting target genes.

The dataset belonging to these two diseases is commensurate since the study's resistant lines were produced on the same susceptible genetic background. The difference in miRNA profiles were imparted by the two $R$-genes and infections of two different Puccinia species. Though the exact mechanism of selective cues for generation of both guide strand and passenger strand of novel-Tae-miR02 from distinct sub-genomes in the fungal and mock inoculated stem rust resistant NIL was unknown, the difference was speculated on account of the $P$. graminis infection. Surprisingly, a variant of the above-mentioned passenger strand originated from same sub-genomal location acts on the interaction among $P$. triticina and the resistant cultivar. Further analysis revealed that the two sub-genomal origin supplemented variation in nucleotides between novel-Tae-miR02 precursor sequences of the corresponding origin points to a common evolutionary origin and refers to to the inductive cues to resistance interaction of stem rust and leaf rust diseases. A common gene family as target for the three forms of novel-Tae-miR02was expansin, which primarily involved in the loosening of plant cell walls[89].

Moreover, their involvement in growth, development, reproduction, ripening, responses to abiotic and biotic stresses, closing and opening of stomata etc., were also characterised[90]. The suppression of expansin genes in rice was reported to contribute to enhanced resistance to bacterial blight disease, which was achieved by improving physical protection by preventing cell wall loosening[91]. Similarly, a mutant line of Atexpla2, an expansin, displayed an enhanced resistance response to Alternaria brassicicola, a necrotrophic fungus[92]. Targeting these cell wall loosening proteins by the novel miRNAs in the rust resistant plants may count as an additional physiological defense response. In addition, the co-occurrence of novel-Tae-miR04 with novel-Tae-miR02-3p of SRM and that of with iso-novel-Tae-miR02$3 p$ in LRI were noticeable irrespective of the various sub-genomal origin. This either represents a specific signal induced miRNA generation and regulation or due to their co-functionality. If not, any of these miRNAs may act as an upstream signal to the other. Further characterisation of these novel miRNAs can pinpoint the so far unknown rust resistance mechanism in future.

\section{Conclusions}

The present investigation identified twenty-six known and seven novel stem rust responsive miRNAs and twenty-two known and four novel leaf rust responsive miRNAs during resistant and susceptible interactions. The identified miRNAs include guide strands, passenger strands and variants of miRNAs in high abundance. Discerning differentially expressed miRNAs and their predicted targets suggest their role in cellular processes, metabolic activities, disease susceptibility and resistance responses. The temporal expression studies identified miRNAs that possibly have regulatory functions over various rust infection stages. The data suggests novel-Tae-miR02 as an important hub in bridging the stem rust and leaf rust 
resistance of bread wheat with its multiple forms. This research work will serve as an important resource to understand miRNA's regulatory role in the two-rust disease management and a reference for miRNA dynamics downstream to the $R$-gene mediated response.

\section{Declarations}

\section{Acknowledgements}

The authors would like to acknowledge Dr. Rebekah Nisha, Dr. Shajitha P and other staffs of ICAR-Indian Agricultural Research Institute, Wellington, for their help in pathogen inoculation and plant material collection. The authors would like to thank the Central University of Kerala for its research facilities.

\section{Disclosure of conflict of interest}

None.

\section{Author contributions}

Minu M Nair: Conceptualization, Methodology, Investigation, Software, Validation, Data curation, Formal analysis, Visualization, Writing- Original draft preparation. Harikrishna Kumar: Software, Data Curation, Formal analysis, Visualization. Jyothsna S: Data curation, Validation, Visualization, Writing- Reviewing and Editing. Krishna T S: Software. Manjunatha C: Resources, Methodology, Writing- Reviewing and Editing. Sivasamy M: Resources, Writing- Reviewing and Editing. Alagu Manickavelu: Conceptualization, Supervision, Writing- Reviewing and Editing.

\section{Financial support}

This research did not receive any specific grant from funding agencies in the public, commercial, or notfor-profit sectors. MMN, and JS are supported by DST-INSPIRE fellowship and CSIR-UGC fellowship, respectively.

\section{References}

[1] J.A. Kolmer, X. Chen, Y. Jin, Diseases which challenge global wheat production-the cereal rusts, Wheat Sci. Trade. (2009) 89-124.

[2] S.C. Bhardwaj, G.P. Singh, O.P. Gangwar, P. Prasad, S. Kumar, Status of wheat rust research and progress in rust management-indian context, Agronomy. 9 (2019) 892.

[3] M.A. Marsalis, N.P. Goldberg, Leaf, stem and stripe rust diseases of wheat. Guide A-415, (2011).

[4] C. Hiebert, W. Spielmeyer, C. McCartney, M. Kassa, T. Fetch, F. You, J. Menzies, G. Humphreys, B. McCallum, Stem rust resistance: two approaches, in: Adv. Wheat Genet. From Genome to F., Springer, Tokyo, 2015: pp. 183-191. 
[5] D. Narang, S. Kaur, B. Steuernagel, S. Ghosh, U. Bansal, J. Li, P. Zhang, S. Bhardwaj, C. Uauy, B.B.H. Wulff, P. Chhuneja, Discovery and characterisation of a new leaf rust resistance gene introgressed in wheat from wild wheat Aegilops peregrina, Sci. Rep. 10 (2020) 1-9.

[6] S. Periyannan, R.J. Milne, M. Figueroa, E.S. Lagudah, P.N. Dodds, An overview of genetic rust resistance: from broad to specific mechanisms, PLoS Pathog. 13 (2017) e1006380.

[7] J.G. Ellis, E.S. Lagudah, W. Spielmeyer, P.N. Dodds, The past, present and future of breeding rust resistant wheat, Front. Plant Sci. 5 (2014) 641.

[8] H.S. Bakala, K.S. Mandahal, L.K. Sarao, P. Srivastava, et al., Breeding Wheat for Biotic Stress Resistance: Achievements, Challenges and Prospects, in: Curr. Trends Wheat Res., IntechOpen, 2021.

[9] K.E. Hammond-Kosack, J.D. Jones, Resistance gene-dependent plant defense responses., Plant Cell. 8 (1996) 1773.

[10] S.T. Chisholm, G. Coaker, B. Day, B.J. Staskawicz, Host-microbe interactions: shaping the evolution of the plant immune response, Cell. 124 (2006) 803-814.

[11] P.-P. Liu, S. Bhattacharjee, D.F. Klessig, P. Moffett, Systemic acquired resistance is induced by $R$ gene-mediated responses independent of cell death, Mol. Plant Pathol. 11 (2010) 155-160.

[12] O.A. Baranova, I.F. Lapochkina, A. V Anisimova, N.R. Gajnullin, I. V lordanskaya, I.Y. Makarova, Identification of Srgenes in new common wheat sources of resistance to stem rust race Ug99 using molecular markers, Russ. J. Genet. Appl. Res. 6 (2016) 344-350.

[13] S. Periyannan, J. Moore, M. Ayliffe, U. Bansal, X. Wang, L. Huang, K. Deal, M. Luo, X. Kong, H. Bariana, et al., The gene Sr33, an ortholog of barley Mla genes, encodes resistance to wheat stem rust race Ug99, Science (80-. ). 341 (2013) 786-788.

[14] B.K. Naik, J.B. Sharma, M. Sivasamy, K. V Prabhu, R.S. Tomar, S.M.S. Tomar, et al., Molecular mapping and validation of the microsatellite markers linked to the Secale cereale-derived leaf rust resistance gene $L r 45$ in wheat, Mol. Breed. 35 (2015) 1-10.

[15] D.P. Cherukuri, S.K. Gupta, A. Charpe, S. Koul, K.V. Prabhu, R.B. Singh, Q.M.R. Haq, Molecular mapping of Aegilops speltoides derived leaf rust resistance gene Lr28 in wheat, Euphytica. 143 (2005) $19-26$.

[16] D. Koujalagi, S.A. Desai, S. Biradar, B.S. Savitha, K.J. Kumar, R. Naik, T.N. Satish, Genetic enhancement of leaf rust resistance in bread wheat (Triticum aestivum L.) through marker-assisted introgression of LR24 and LR28, Appl. Biol. Res. 21 (2019) 1-9.

[17] A.T. Djami-Tchatchou, N. Sanan-Mishra, K. Ntushelo, I.A. Dubery, Functional Roles of microRNAs in Agronomically Important Plants-Potential as Targets for Crop Improvement and Protection, Front. Plant 
Sci. 8 (2017) 378. https://doi.org/10.3389/fpls.2017.00378.

[18] M.M. Nair, A. Manickavelu, MicroRNAs as fine-tuners of gene regulation in plant-microbe interactions, Curr. Sci. 119 (2020) 1282-1290.

[19] P.K. Gupta, MicroRNAs and target mimics for crop improvement, Curr. Sci. (2015) 1624-1633.

[20] S. Campo, C. Peris-Peris, C. Siré, A.B. Moreno, L. Donaire, M. Zytnicki, C. Notredame, C. Llave, B. San Segundo, Identification of a novel micro RNA (miRNA) from rice that targets an alternatively spliced transcript of the Nramp6 (Natural resistance-associated macrophage protein 6) gene involved in pathogen resistance, New Phytol. 199 (2013) 212-227.

[21] Á.L. Pérez-Quintero, A. Quintero, O. Urrego, P. Vanegas, C. López, Bioinformatic identification of cassava miRNAs differentially expressed in response to infection by Xanthomonas axonopodis pv. manihotis, BMC Plant Biol. 12 (2012) 1-11.

[22] H. Budak, B.A. Akpinar, Plant miRNAs: biogenesis, organization and origins, Funct. \\& Integr. Genomics. 15 (2015) 523-531.

[23] O.P. Gupta, V. Permar, V. Koundal, U.D. Singh, S. Praveen, MicroRNA regulated defense responses in Triticum aestivum L. during Puccinia graminis f. sp. tritici infection, Mol. Biol. Rep. 39 (2012) 817-824.

[24] D. Kumar, S. Dutta, D. Singh, K.V. Prabhu, M. Kumar, K. Mukhopadhyay, Uncovering leaf rust responsive miRNAs in wheat (Triticum aestivum $\mathrm{L}$.) using high-throughput sequencing and prediction of their targets through degradome analysis, Planta. 245 (2017) 161-182.

[25] D. Singh, G. Bhaganagare, R. Bandopadhyay, K.V. Prabhu, P.K. Gupta, K. Mukhopadhyay, Targeted spatio-temporal expression based characterization of state of infection and time-point of maximum defense in wheat NILs during leaf rust infection, Mol. Biol. Rep. 39 (2012) 9373-9382.

[26] M. Martin, Cutadapt removes adapter sequences from high-throughput sequencing reads, EMBnet. J. 17 (2011) 10-12.

[27] M. Alaux, J. Rogers, T. Letellier, R. Flores, F. Alfama, C. Pommier, N. Mohellibi, S. Durand, E. Kimmel, C. Michotey, C. Guerche, M. Loaec, M. Lainé, D. Steinbach, F. Choulet, H. Rimbert, P. Leroy, N. Guilhot, J. Salse, C. Feuillet, E. Paux, K. Eversole, A.-F. Adam-Blondon, H. Quesneville, I.W.G.S. Consortium, Linking the International Wheat Genome Sequencing Consortium bread wheat reference genome sequence to wheat genetic and phenomic data, Genome Biol. 19 (2018) 111. https://doi.org/10.1186/s13059-0181491-4.

[28] B. Langmead, Aligning short sequencing reads with Bowtie, Curr. Protoc. Bioinforma. 32 (2010) 1117. 
[29] M.R. Friedländer, S.D. Mackowiak, N. Li, W. Chen, N. Rajewsky, miRDeep2 accurately identifies known and hundreds of novel microRNA genes in seven animal clades, Nucleic Acids Res. 40 (2012) 3752.

[30] S. Griffiths-Jones, R.J. Grocock, S. van Dongen, A. Bateman, A.J. Enright, miRBase: microRNA sequences, targets and gene nomenclature, Nucleic Acids Res. 34 (2006) D140-D144. https://doi.org/10.1093/nar/gkj112.

[31] M.J. Axtell, B.C. Meyers, Revisiting criteria for plant microRNA annotation in the era of big data, Plant Cell. 30 (2018) 272-284.

[32] K. Yang, G. Sablok, G. Qiao, Q. Nie, X. Wen, isomiR2Function: an integrated workflow for identifying microRNA variants in plants, Front. Plant Sci. 8 (2017) 322.

[33] I.A. Shahmuradov, R.K. Umarov, V. V Solovyev, TSSPlant: a new tool for prediction of plant Pol II promoters, Nucleic Acids Res. 45 (2017) e65--e65.

[34] M. Lescot, P. Déhais, G. Thijs, K. Marchal, Y. Moreau, Y. de Peer, P. Rouzé, S. Rombauts, PlantCARE, a database of plant cis-acting regulatory elements and a portal to tools for in silico analysis of promoter sequences, Nucleic Acids Res. 30 (2002) 325-327.

[35] S. Temnykh, G. DeClerck, A. Lukashova, L. Lipovich, S. Cartinhour, S. McCouch, Computational and experimental analysis of microsatellites in rice (Oryza sativa L.): frequency, length variation, transposon associations, and genetic marker potential, Genome Res. 11 (2001) 1441-1452.

[36] M.I. Love, W. Huber, S. Anders, Moderated estimation of fold change and dispersion for RNA-seq data with DESeq2, Genome Biol. 15 (2014) 1-21.

[37] J. Goedhart, M.S. Luijsterburg, VolcaNoseR is a web app for creating, exploring, labeling and sharing volcano plots, Sci. Rep. 10 (2020) 20560. https://doi.org/10.1038/s41598-020-76603-3.

[38] X. Dai, Z. Zhuang, P.X. Zhao, psRNATarget: a plant small RNA target analysis server (2017 release), Nucleic Acids Res. 46 (2018) W49-W54.

[39] A. Conesa, S. Götz, Blast2GO: a comprehensive suite for functional analysis in plant genomics, Int. J. Plant Genomics. 2008 (2008).

[40] L. Chen, Y.-H. Zhang, M. Zheng, T. Huang, Y.-D. Cai, Identification of compound-protein interactions through the analysis of gene ontology, KEGG enrichment for proteins and molecular fragments of compounds, Mol. Genet. Genomics. 291 (2016) 2065-2079.

[41] D. Szklarczyk, A. Franceschini, S. Wyder, K. Forslund, D. Heller, J. Huerta-Cepas, M. Simonovic, A. Roth, A. Santos, K.P. Tsafou, others, STRING v10: protein--protein interaction networks, integrated over the tree of life, Nucleic Acids Res. 43 (2015) D447-D452. 
[42] E. Varkonyi-Gasic, Stem-loop qRT-PCR for the detection of plant microRNAs, in: Plant Epigenetics, Springer, 2017: pp. 163-175.

[43] A. Untergasser, I. Cutcutache, T. Koressaar, J. Ye, B.C. Faircloth, M. Remm, S.G. Rozen, Primer3-new capabilities and interfaces, Nucleic Acids Res. 40 (2012) e115-e115.

https://doi.org/10.1093/nar/gks596.

[44] K.J. Livak, T.D. Schmittgen, Analysis of Relative Gene Expression Data Using Real-Time Quantitative PCR and the $2^{-\triangle \triangle C T}$ Method, Methods. 25 (2001) 402-408.

https://doi.org/https://doi.org/10.1006/meth.2001.1262.

[45] L.-C. Hsieh, S.-I. Lin, A.C.-C. Shih, J.-W. Chen, W.-Y. Lin, C.-Y. Tseng, W.-H. Li, T.-J. Chiou, Uncovering Small RNA-Mediated Responses to Phosphate Deficiency in Arabidopsis by Deep Sequencing, Plant Physiol. 151 (2009) 2120-2132. https://doi.org/10.1104/pp.109.147280.

[46] B. Liu, G. Sun, microRNAs contribute to enhanced salt adaptation of the autopolyploid Hordeum bulbosum compared with its diploid ancestor, Plant J. 91 (2017) 57-69.

https://doi.org/https://doi.org/10.1111/tpj.13546.

[47] X. Zhang, H. Zhao, S. Gao, W.-C. Wang, S. Katiyar-Agarwal, H.-D. Huang, N. Raikhel, H. Jin, Arabidopsis Argonaute 2 Regulates Innate Immunity via miRNA393*-Mediated Silencing of a GolgiLocalized SNARE Gene, MEMB12, Mol. Cell. 42 (2011) 356-366.

https://doi.org/https://doi.org/10.1016/j.molcel.2011.04.010.

[48] L. Navarro, P. Dunoyer, F. Jay, B. Arnold, N. Dharmasiri, M. Estelle, O. Voinnet, J.D.G. Jones, A Plant miRNA Contributes to Antibacterial Resistance by Repressing Auxin Signaling, Science (80-. ). 312 (2006) 436-439. https://doi.org/10.1126/science.1126088.

[49] G. Sablok, A.K. Srivastva, P. Suprasanna, V. Baev, P.J. Ralph, isomiRs: Increasing Evidences of isomiRs Complexity in Plant Stress Functional Biology, Front. Plant Sci. 6 (2015) 949. https://doi.org/10.3389/fpls.2015.00949.

[50] F. Ahmed, M. Senthil-Kumar, S. Lee, X. Dai, K.S. Mysore, P.X. Zhao, Comprehensive analysis of small RNA-seq data reveals that combination of miRNA with its isomiRs increase the accuracy of target prediction in Arabidopsis thaliana, RNA Biol. 11 (2014) 1414-1429. https://doi.org/10.1080/15476286.2014.996474.

[51] B. Liu, P. Li, X. Li, C. Liu, S. Cao, C. Chu, X. Cao, Loss of Function of OsDCL 1 Affects MicroRNA Accumulation and Causes Developmental Defects in Rice, Plant Physiol. 139 (2005) 296-305. https://doi.org/10.1104/pp.105.063420.

[52] X. Fang, Y. Qi, RNAi in Plants: An Argonaute-Centered View, Plant Cell. 28 (2016) 272-285. https://doi.org/10.1105/tpc.15.00920. 
[53] N. Jain, N. Sinha, H. Krishna, P.K. Singh, T. Gautam, P. Prasad, H.S. Balyan, P.K. Gupta, A study of miRNAs and IncRNAs during $L$ r28-mediated resistance against leaf rust in wheat (Triticum aestivum $L$.), Physiol. Mol. Plant Pathol. 112 (2020) 101552.

https://doi.org/https://doi.org/10.1016/j.pmpp.2020.101552.

[54] Z. Xie, E. Allen, N. Fahlgren, A. Calamar, S.A. Givan, J.C. Carrington, Expression of ArabidopsisMIRNA Genes, Plant Physiol. 138 (2005) 2145-2154.

https://doi.org/10.1104/pp.105.062943.

[55] M. Megraw, V. Baev, V. Rusinov, S.T. Jensen, K. Kalantidis, A.G. Hatzigeorgiou, MicroRNA promoter element discovery in Arabidopsis, RNA. 12 (2006) 1612-1619. https://doi.org/10.1261/rna.130506.

[56] C. Sánchez-Retuerta, P. Suaréz-López, R. Henriques, Under a New Light: Regulation of LightDependent Pathways by Non-coding RNAs, Front. Plant Sci. 9 (2018) 962.

https://doi.org/10.3389/fpls.2018.00962.

[57] S.P. Hazen, F. Naef, T. Quisel, J.M. Gendron, H. Chen, J.R. Ecker, J.O. Borevitz, S.A. Kay, Exploring the transcriptional landscape of plant circadian rhythms using genome tiling arrays, Genome Biol. 10 (2009) R17. https://doi.org/10.1186/gb-2009-10-2-r17.

[58] A. Kaur, P.K. Pati, A.M. Pati, A.K. Nagpal, In-silico analysis of cis-acting regulatory elements of pathogenesis-related proteins of Arabidopsis thaliana and Oryza sativa, PLoS One. 12 (2017) e0184523. https://doi.org/10.1371/journal.pone.0184523.

[59] V. Petrov, V. Vermeirssen, I. De Clercq, F. Van Breusegem, I. Minkov, K. Vandepoele, T.S. Gechev, Identification of cis-regulatory elements specific for different types of reactive oxygen species in Arabidopsis thaliana, Gene. 499 (2012) 52-60.

https://doi.org/https://doi.org/10.1016/j.gene.2012.02.035.

[60] V. Garretón, J. Carpinelli, X. Jordana, L. Holuigue, The as-1 Promoter Element Is an Oxidative StressResponsive Element and Salicylic Acid Activates It via Oxidative Species, Plant Physiol. 130 (2002) 1516-1526. https://doi.org/10.1104/pp.009886.

[61] I. Gadjev, S. Vanderauwera, T.S. Gechev, C. Laloi, I.N. Minkov, V. Shulaev, K. Apel, D. Inzé, R. Mittler, F. Van Breusegem, Transcriptomic Footprints Disclose Specificity of Reactive Oxygen Species Signaling in Arabidopsis, Plant Physiol. 141 (2006) 436-445. https://doi.org/10.1104/pp.106.078717.

[62] Y. Fujita, M. Fujita, K. Shinozaki, K. Yamaguchi-Shinozaki, ABA-mediated transcriptional regulation in response to osmotic stress in plants, J. Plant Res. 124 (2011) 509-525. https://doi.org/10.1007/s10265-011-0412-3.

[63] S.R. McCouch, X. Chen, O. Panaud, S. Temnykh, Y. Xu, Y.G. Cho, N. Huang, T. Ishii, M. Blair, Microsatellite marker development, mapping and applications in rice genetics and breeding BT - Oryza: 
From Molecule to Plant. (1997) 89-99. https://doi.org/10.1007/978-94-011-5794-0_9.

[64] S.H. Garland, L. Lewin, M. Abedinia, R. Henry, A. Blakeney, The use of microsatellite polymorphisms for the identification of Australian breeding lines of rice (Oryza sativa L.), Euphytica. 108 (1999) 53. https://doi.org/10.1023/A:1003688612179.

[65] S. Tyagi, A. Kumar, T. Gautam, R. Pandey, S. Rustgi, R.R. Mir, Development and use of miRNAderived SSR markers for the study of genetic diversity, population structure, and characterization of genotypes for breeding heat tolerant wheat varieties, PLoS One. 16 (2021) 1-17.

https://doi.org/10.1371/journal.pone.0231063.

[66] Y.-F. Li, K. Wei, M. Wang, L. Wang, J. Cui, D. Zhang, J. Guo, M. Zhao, Y. Zheng, Identification and Temporal Expression Analysis of Conserved and Novel MicroRNAs in the Leaves of Winter Wheat Grown in the Field, Front. Genet. 10 (2019) 779. https://doi.org/10.3389/fgene.2019.00779.

[67] M. Roberti, P.L. Polosa, F. Bruni, C. Manzari, S. Deceglie, M.N. Gadaleta, P. Cantatore, The MTERF family proteins: Mitochondrial transcription regulators and beyond, Biochim. Biophys. Acta - Bioenerg. 1787 (2009) 303-311. https://doi.org/https://doi.org/10.1016/j.bbabio.2009.01.013.

[68] V. Quesada, The roles of mitochondrial transcription termination factors (MTERFs) in plants, Physiol. Plant. 157 (2016) 389-399. https://doi.org/https://doi.org/10.1111/ppl.12416.

[69] R. Karlova, J.C. van Haarst, C. Maliepaard, H. van de Geest, A.G. Bovy, M. Lammers, G.C. Angenent, R.A. de Maagd, Identification of microRNA targets in tomato fruit development using high-throughput sequencing and degradome analysis, J. Exp. Bot. 64 (2013) 1863-1878.

https://doi.org/10.1093/jxb/ert049.

[70] F. Gao, K. Wang, Y. Liu, Y. Chen, P. Chen, Z. Shi, J. Luo, D. Jiang, F. Fan, Y. Zhu, S. Li, Blocking miR396 increases rice yield by shaping inflorescence architecture, Nat. Plants. 2 (2015) 15196. https://doi.org/10.1038/nplants.2015.196.

[71] K. Zhang, X. Shi, X. Zhao, D. Ding, J. Tang, J. Niu, Investigation of miR396 and growth-regulating factor regulatory network in maize grain filling, Acta Physiol. Plant. 37 (2015) 28. https://doi.org/10.1007/s11738-014-1767-6.

[72] M.A. Omidbakhshfard, S. Proost, U. Fujikura, B. Mueller-Roeber, Growth-Regulating Factors (GRFs): A Small Transcription Factor Family with Important Functions in Plant Biology, Mol. Plant. 8 (2015) 9981010. https://doi.org/https://doi.org/10.1016/j.molp.2015.01.013.

[73] M. Soto-Suárez, P. Baldrich, D. Weigel, I. Rubio-Somoza, B. San Segundo, The Arabidopsis miR396 mediates pathogen-associated molecular pattern-triggered immune responses against fungal pathogens, Sci. Rep. 7 (2017) 44898. https://doi.org/10.1038/srep44898. 
[74] P.-P. Liu, T.A. Montgomery, N. Fahlgren, K.D. Kasschau, H. Nonogaki, J.C. Carrington, Repression of AUXIN RESPONSE FACTOR10 by microRNA160 is critical for seed germination and post-germination stages, Plant J. 52 (2007) 133-146. https://doi.org/https://doi.org/10.1111/j.1365-313X.2007.03218.x.

[75] J.-W. Wang, L.-J. Wang, Y.-B. Mao, W.-J. Cai, H.-W. Xue, X.-Y. Chen, Control of Root Cap Formation by MicroRNA-Targeted Auxin Response Factors in Arabidopsis, Plant Cell. 17 (2005) 2204-2216. https://doi.org/10.1105/tpc.105.033076.

[76] A.C. Mallory, D.P. Bartel, B. Bartel, MicroRNA-Directed Regulation of Arabidopsis AUXIN RESPONSE FACTOR17 Is Essential for Proper Development and Modulates Expression of Early Auxin Response Genes, Plant Cell. 17 (2005) 1360-1375. https://doi.org/10.1105/tpc.105.031716.

[77] B. Natarajan, H.S. Kalsi, P. Godbole, N. Malankar, A. Thiagarayaselvam, S. Siddappa, H. V Thulasiram, S.K. Chakrabarti, A.K. Banerjee, MiRNA160 is associated with local defense and systemic acquired resistance against Phytophthora infestans infection in potato, J. Exp. Bot. 69 (2018) 20232036. https://doi.org/10.1093/jxb/ery025.

[78] A. Burkhardt, B. Day, Transcriptome and Small RNAome Dynamics during a Resistant and Susceptible Interaction between Cucumber and Downy Mildew, Plant Genome. 9 (2016) plantgenome2015.08.0069. https://doi.org/https://doi.org/10.3835/plantgenome2015.08.0069.

[79] Z. Chen, A Superfamily of Proteins with Novel Cysteine-Rich Repeats, Plant Physiol. 126 (2001) 473-476. https://doi.org/10.1104/pp.126.2.473.

[80] M. Wrzaczek, M. Brosché, J. Salojärvi, S. Kangasjärvi, N. Idänheimo, S. Mersmann, S. Robatzek, S. Karpiński, B. Karpińska, J. Kangasjärvi, Transcriptional regulation of the CRK/DUF26 group of Receptorlike protein kinases by ozone and plant hormones in Arabidopsis, BMC Plant Biol. 10 (2010) 95. https://doi.org/10.1186/1471-2229-10-95.

[81] Y.-H. Yeh, Y.-H. Chang, P.-Y. Huang, J.-B. Huang, L. Zimmerli, Enhanced Arabidopsis pattern-triggered immunity by overexpression of cysteine-rich receptor-like kinases, Front. Plant Sci. 6 (2015) 322. https://doi.org/10.3389/fpls.2015.00322.

[82] J. Gu, J. Sun, N. Liu, X. Sun, C. Liu, L. Wu, G. Liu, F. Zeng, C. Hou, S. Han, W. Zhen, D. Wang, A novel cysteine-rich receptor-like kinase gene, TaCRK2, contributes to leaf rust resistance in wheat, Mol. Plant Pathol. 21 (2020) 732-746. https://doi.org/https://doi.org/10.1111/mpp.12929.

[83] S. Fujimoto, S. Matsunaga, M. Yonemura, S. Uchiyama, T. Azuma, K. Fukui, Identification of a novel plant MAR DNA binding protein localized on chromosomal surfaces, Plant Mol. Biol. 56 (2004) 225-239. https://doi.org/10.1007/s11103-004-3249-5.

[84] H. Lu, Y. Zou, N. Feng, Overexpression of AHL20 Negatively Regulates Defenses in Arabidopsis, J. Integr. Plant Biol. 52 (2010) 801-808. https://doi.org/https://doi.org/10.1111/j.1744-7909.2010.00969.x. 
[85] Y. Zhang, R. Xia, H. Kuang, B.C. Meyers, The Diversification of Plant NBS-LRR Defense Genes Directs the Evolution of MicroRNAs That Target Them, Mol. Biol. Evol. 33 (2016) 2692-2705. https://doi.org/10.1093/molbev/msw154.

[86] Y. Li, Q. Zhang, J. Zhang, L. Wu, Y. Qi, J.-M. Zhou, Identification of MicroRNAs Involved in PathogenAssociated Molecular Pattern-Triggered Plant Innate Immunity, Plant Physiol. 152 (2010) 2222-2231. https://doi.org/10.1104/pp.109.151803.

[87] X. Wang, B.D. McCallum, T. Fetch, G. Bakkeren, B.J. Saville, Sr36-and Sr5-Mediated Resistance Response to Puccinia graminis f. sp. tritici Is Associated with Callose Deposition in Wheat Guard Cells, Phytopathology®. 105 (2015) 728-737. https://doi.org/10.1094/PHYTO-08-14-0213-R.

[88] G. Hu, F.H.J. Rijkenberg, Scanning electron microscopy of early infection structure formation by Puccinia recondita f. sp. tritici on and in susceptible and resistant wheat lines, Mycol. Res. 102 (1998) 391-399. https://doi.org/https://doi.org/10.1017/S0953756297005054.

[89] D.J. Cosgrove, Plant expansins: diversity and interactions with plant cell walls, Curr. Opin. Plant Biol. 25 (2015) 162-172. https://doi.org/https://doi.org/10.1016/j.pbi.2015.05.014.

[90] P.-C. Wei, X.-Q. Zhang, P. Zhao, X.-C. Wang, Regulation of stomatal opening by the guard cell expansin AtEXPA1, Plant Signal. Behav. 6 (2011) 740-742. https://doi.org/10.4161/psb.6.5.15144.

[91] W. Li, F. Wang, J. Wang, F. Fan, J. Zhu, J. Yang, F. Liu, W. Zhong, Overexpressing CYP71Z2 enhances resistance to bacterial blight by suppressing auxin biosynthesis in rice, PLoS One. 10 (2015) e0119867e0119867. https://doi.org/10.1371/journal.pone.0119867.

[92] S. Abuqamar, S. Ajeb, A. Sham, M.R. Enan, R. Iratni, A mutation in the expansin-like A2 gene enhances resistance to necrotrophic fungi and hypersensitivity to abiotic stress in Arabidopsis thaliana, Mol. Plant Pathol. 14 (2013) 813-827. https://doi.org/https://doi.org/10.1111/mpp.12049.

\section{Figures}



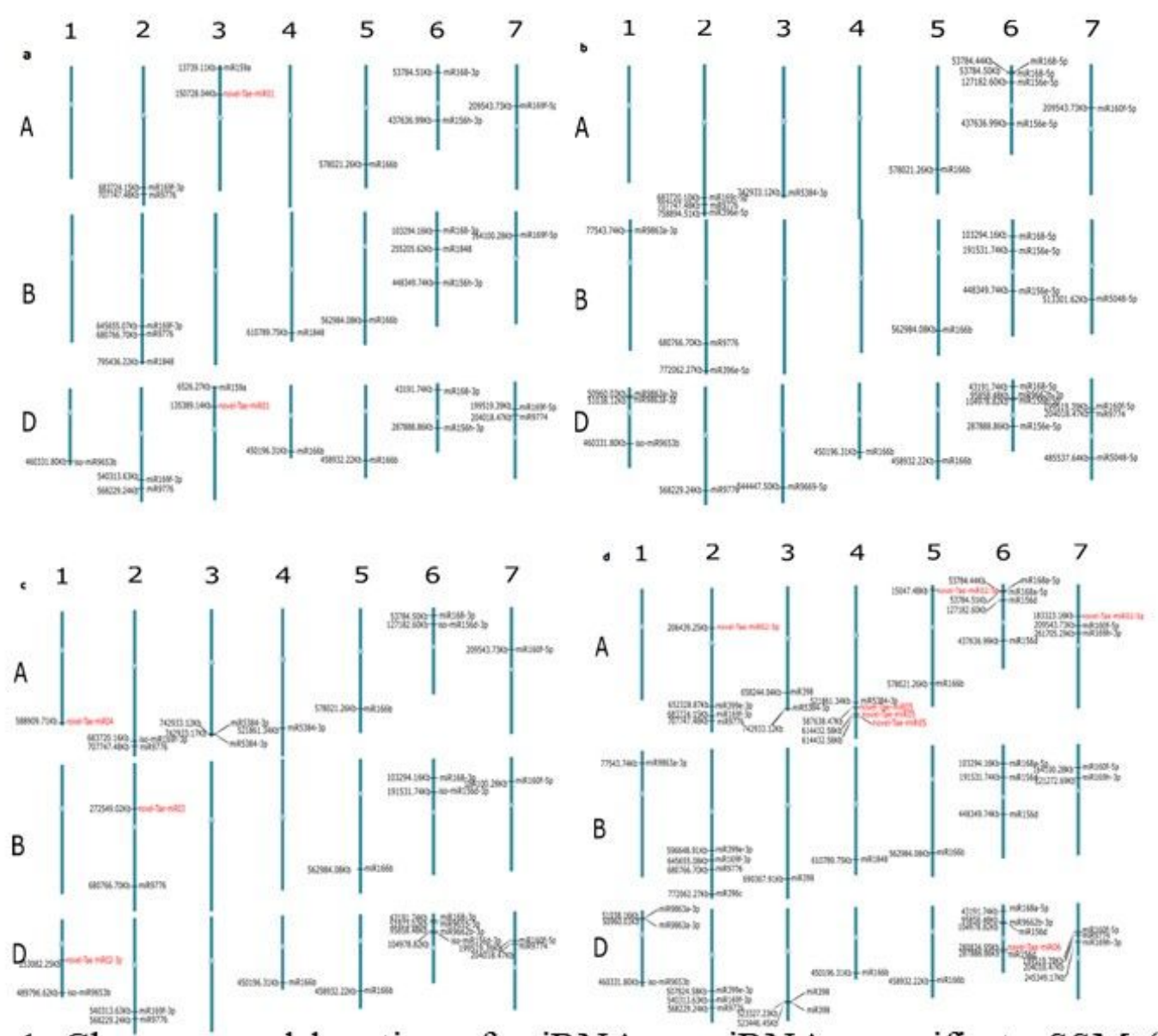

Figure 1. Chromosomal location of miRNAs. miRNAs specific to SSM (a), SSI (b), SRM (c) and SRI (d) treated libraries are depicted. Wheat chromosomes are represented as vertical bars. The miRNAs are labelled on the right side (novel miRNAs in red) and their respective positions are labelled on the left side of the chromosome.

\section{Figure 1}

Chromosomal location of miRNAs. miRNAs specific to SSM (a), SSI (b), SRM (c) and SRI (d) treated libraries are depicted. Wheat chromosomes are represented as vertical bars. The miRNAs are labelled on the right side (novel miRNAs in red) and their respective positions are labelled on the left side of the chromosome. 

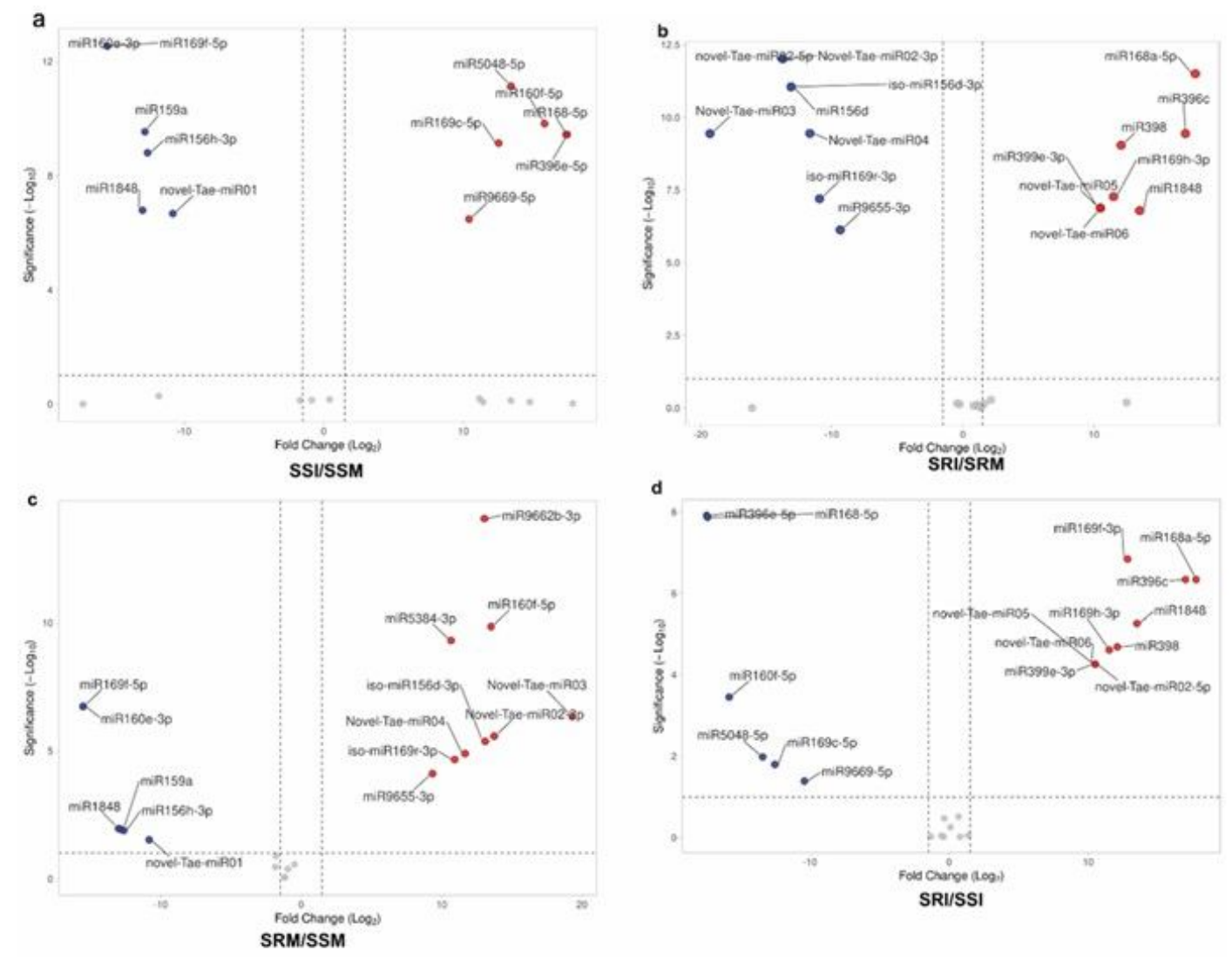

Figure 2. Volcano plot showing differentially expressed miRNAs between stem rust inoculation treatments. The upregulated and downregulated miRNAs in the libraries are represented in red and blue colour respectively. miRNAs with no significant fold change are represented in green.

\section{Figure 2}

Volcano plot showing differentially expressed miRNAs between stem rust inoculation treatments. The upregulated and downregulated miRNAs in the libraries are represented in red and blue colour respectively. miRNAs with no significant fold change are represented in green. 

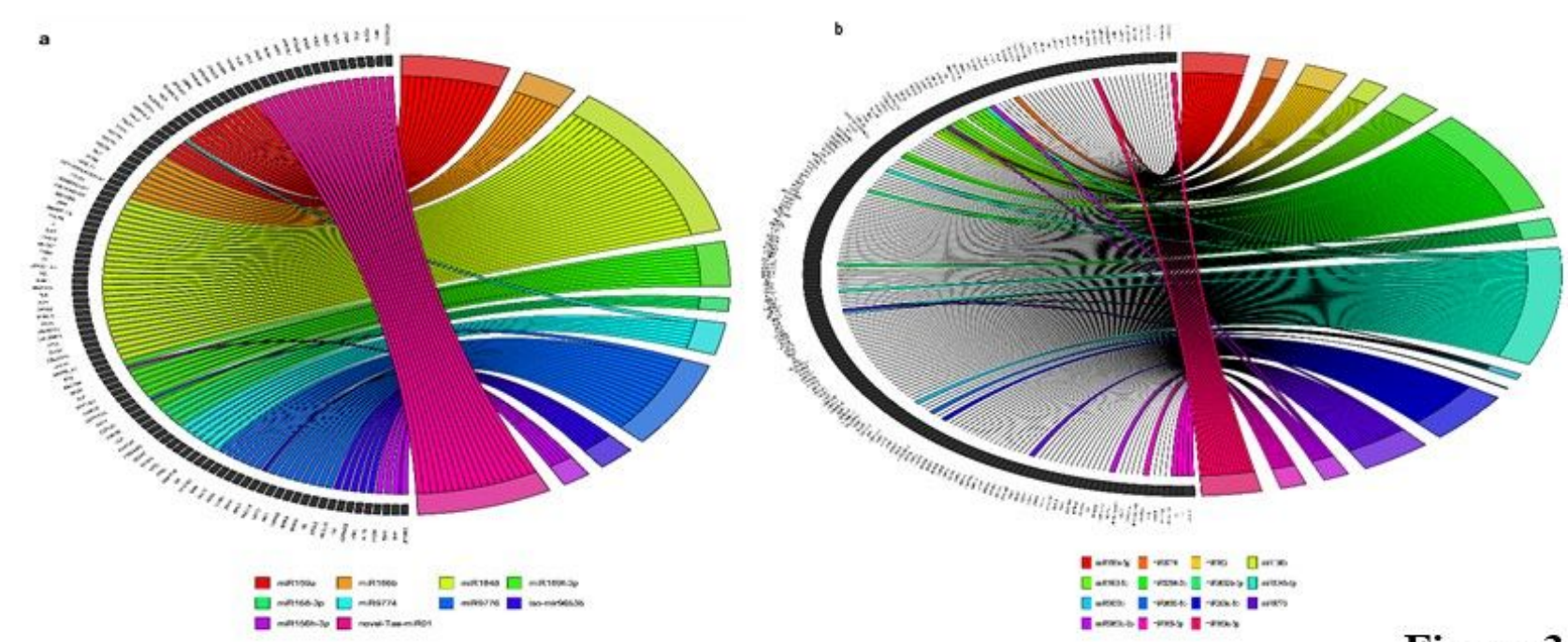

Figure 3.
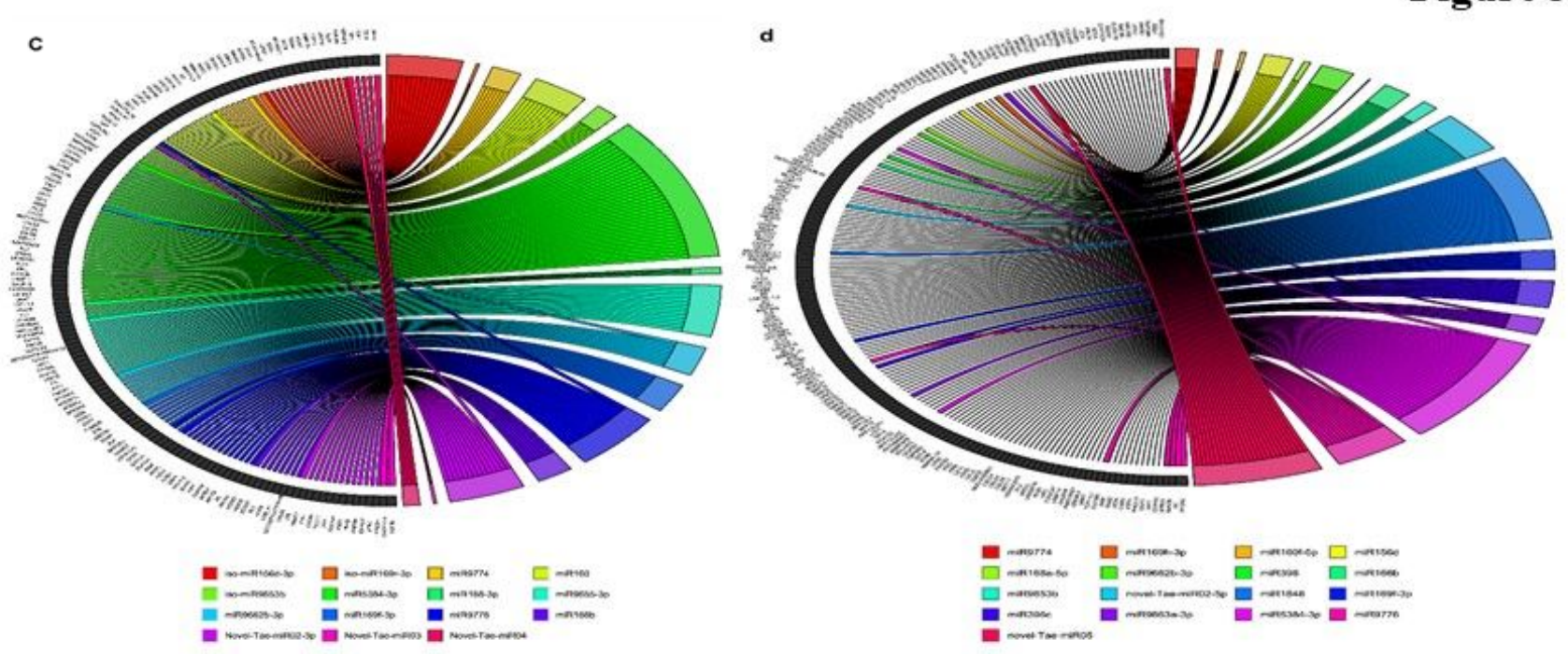

Figure 3

Target genes predicted for stem rust responsive wheat miRNAs. Targets of miRNAs in SSM (a), SSI (b), SRM (c) and SRI (d) libraries are shown. Each miRNA represented in color bars in the right side are linked to multiple targets depicted in the left side of the circos plot. 
Figure 4.
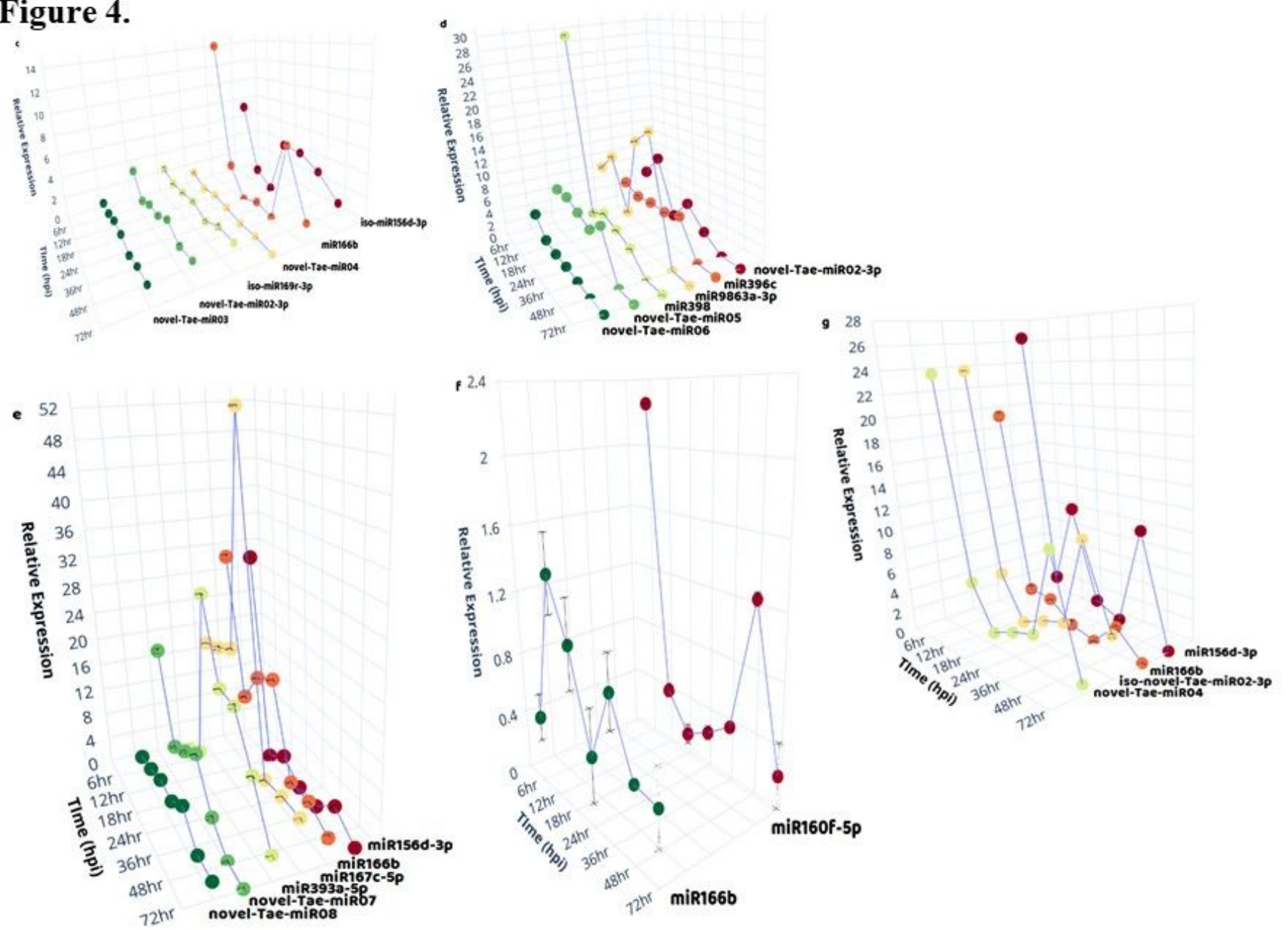

Figure 4

Temporal differential expression of miRNAs associated with stem rust and leaf rust diseases. From each library, mature miRNAs were selected and relative expression was obtained from real-time PCR data. Relative expression of miRNAs in SSM/ LSM (a), SSI (b), SRM (c), SRI (d), LSI (e), LRM (f), LRI (g) are represented as $3 \mathrm{D}$ plot. Expression values are expressed as the ratio of $2-\Delta \Delta C T$ relative to the control sample 0 hpi and internal reference U6 RNA. 